\title{
2 Kinetically controlling the length of self-assembled polymer 3 nanofibers formed by intermolecular hydrogen bonds
}

4 Franka Gruschwitz, ${ }^{a, b, \neq}$ Tobias Klein, ${ }^{a, b, \neq}$ Maren T. Kuchenbrod, ${ }^{a, b}$ Naoto Moriyama, ${ }^{c}$ Shota Fujii, ${ }^{c}$

5 Ivo Nischang,a,b Stephanie Hoeppener,a,b Kazuo Sakurai,c Ulrich S. Schubert,a,b Johannes C.

6 Brendel, $, \mathrm{a}, *$

7 a Laboratory of Organic and Macromolecular Chemistry (IOMC), Friedrich Schiller University

8 Jena, Humboldtstraße 10, 07743 Jena, Germany

9 b Jena Center for Soft Matter (JCSM), Friedrich Schiller University Jena, Philosophenweg 7, 1007743 Jena, Germany

11 c Department of Chemistry and Biochemistry, University of Kitakyushu, 1-1 Hibikino, 12 Wakamatsu-ku, Kitakyushu, Fukuoka 808-0135, Japan

13

$14 \neq$ These authors contributed equally to this work

$15 *$ corresponding author: johannes.brendel@uni-je na.de

16 


\section{Content}

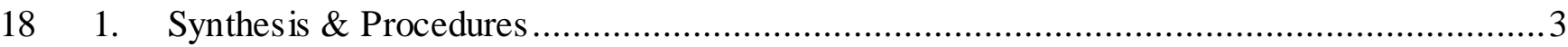

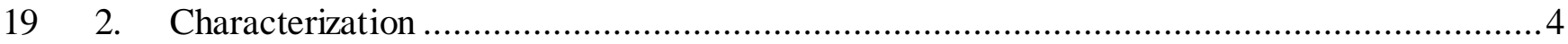

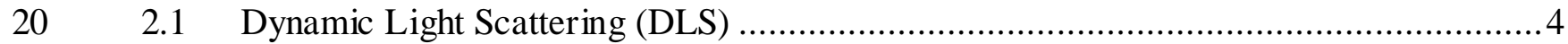

212.2 CryoTrans mission Electron Microscopy (cryoTEM) ......................................

$22 \quad 2.3$ Asymmetrical Flow Field-Flow Fractionation (AF4) ...................................... 15

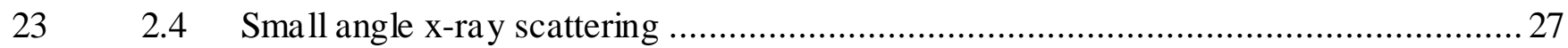

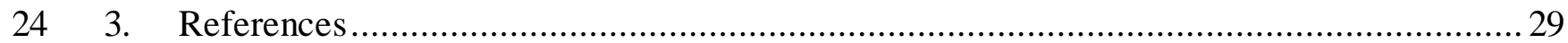




\section{1. Synthesis \& Procedures}

28 Materials and Methods. All reagents and solvents were commercial products purchased from

29 Sigma-Aldrich, abcr, Iris BioTech, Rapp Polymere or TCI and were used without further

30 purification.

\section{Synthesis.}

32 The benzenetrisurea-polyethylene oxide (BTU) and benzenetrispeptide-polyethyle ne oxide (BTP)

33 conjugates were synthesized according to previously published protocols. ${ }^{1-2}$

\section{Self-as sembly procedures.}

\section{Solvent switch method}

$365 \mathrm{mg}$ of B TU or B TP were dissolved in $1 \mathrm{~mL}$ of the respective organic solvent (DMF, THF, acetone

37 or ethanol) and stirred overnight to guarantee complete dissolution. To this, $4 \mathrm{~mL}$ of MilliQ water 38 were added at the specified speed ( 1 to $\left.100 \mathrm{ml} \mathrm{h}^{-1}\right)$ using a syringe pump under vigorous stirring to 39 reach a final water content of $80 \mathrm{v} \%$. For this purpose, the needle of the syringe was immersed in

40 the organic BTP or BTU solution to enable a constant release of MilliQ water from the syringe 41 and avoid the formation of drops that would result in high local water concentrations at the spot 42 where the drop immerses into the solution. Afterwards, the solution was transferred to float-a43 lyzer® tubings with a molecular weight cutoff of $3.5 \mathrm{kD}$ and dialyzed for five days against water 44 to remove all organic solvent traces.

\section{Quenching method}

$465 \mathrm{mg}$ of B TU or B TP were dissolved in $1 \mathrm{~mL}$ of the respective organic solvent (DMF, THF, acetone 47 or ethanol) and stirred overnight to guarantee complete dissolution. This organic solution was 48 added to $4 \mathrm{~mL}$ MilliQ water using a syringe pump $\left(1 \mathrm{~mL} \mathrm{~h}^{-1}\right)$ under vigorous stirring to reach a 49 final water content of $80 \mathrm{v} \%$. For this purpose, the needle of the syringe was immersed in the MilliQ 50 water to enable a constant release of organic B TP or BTU solution from the syringe and avoid the

51 formation of drops that would result in high local BTP or BTU solution concentrations at the spot 52 where the drop immerses into the solution. Afterwards, the solution was transferred to float-a53 lyzer® tubings with a molecular weight cutoff of $3.5 \mathrm{kD}$ and dialyzed for five days against water 54 to remove all organic solvent traces. 


\section{2. Characterization}

\section{$56 \quad 2.1$ Dynamic Light Scattering (DLS)}

57 A scattering angle of $173^{\circ}$ was used to record intensity fluctuations of the different samples in 58 solution. All measurements were conducted in triplicate at a temperature of $25{ }^{\circ} \mathrm{C}$ in disposable

59 macro cuvettes containing $2 \mathrm{~mL}$ solution and after allowing for an equilibration time of $60 \mathrm{~s}$. The 60 acquisition time was $60 \mathrm{~s}$. The apparent distribution of intensity-weighted hydrodynamic radii, $d_{h}$, 61 was obtained from the Stokes-Einstein equation:

$$
d_{h}=\frac{k T}{3 \pi \eta D}
$$

63 with $k$ being the Boltzmann constant, $T$ the temperature in units $\mathrm{K}, \eta$ the viscosity of the solvent, 64 and $D$ the apparent translational diffusion coefficient at the utilized concentrations.

65 For DLS measurements of solvent mixtures the water was added manually using pipettes unless 66 stated otherwise. 

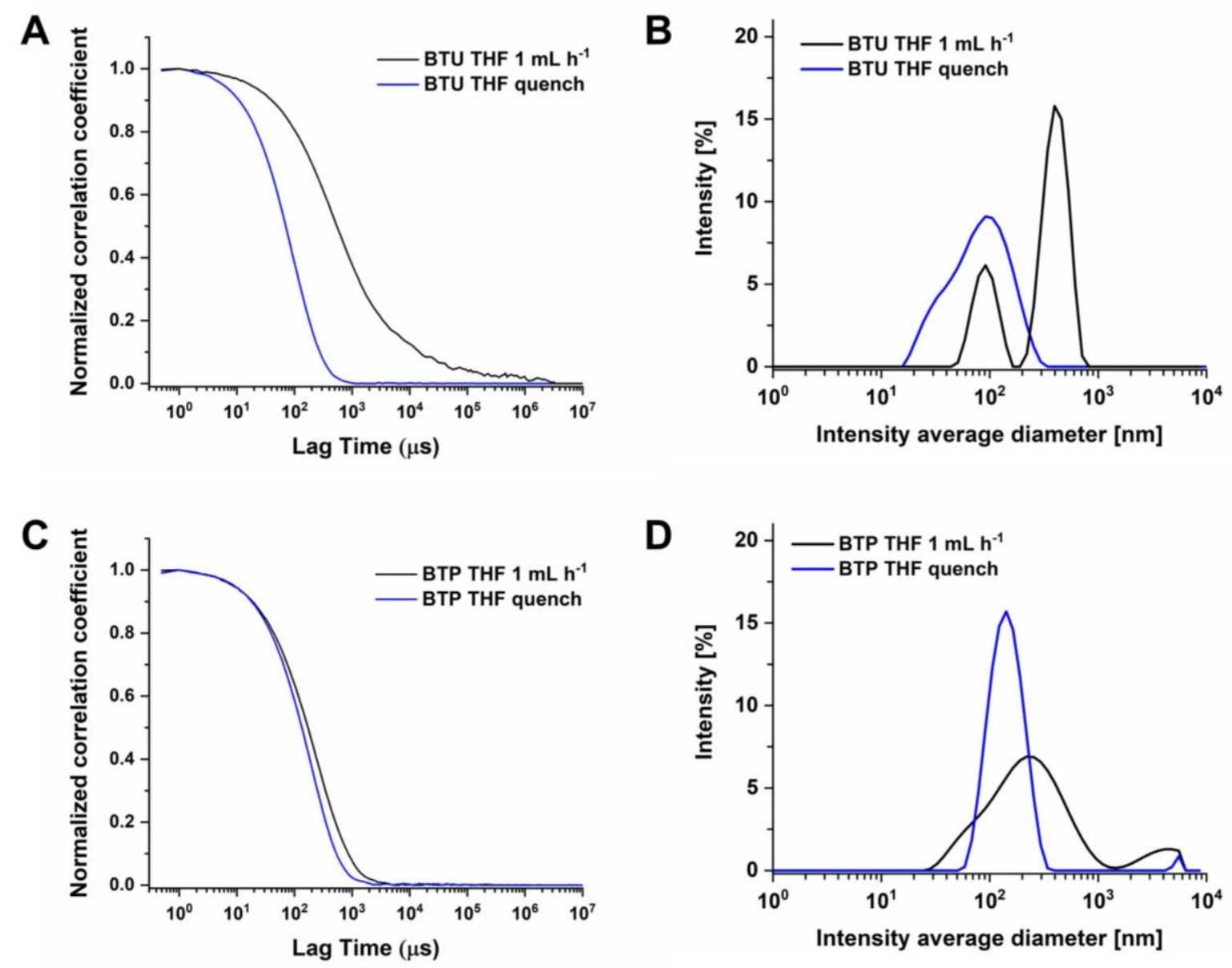

Figure S1: DLScorrelograms of BTU (A) and BTP (B) in water for the solvent switch (THF $1 \mathbf{~ m L ~ h}^{-1}$; black) and the quenching 69 procedure (THF quench; blue). Correlograms were recorded in MilliQ water at a concentration of $1 \mathrm{mg} \mathrm{mL}^{-1}$. The corresponding 70 intensity average diameters for BTU and B TP can be seen in (C) and (D), respectively. 

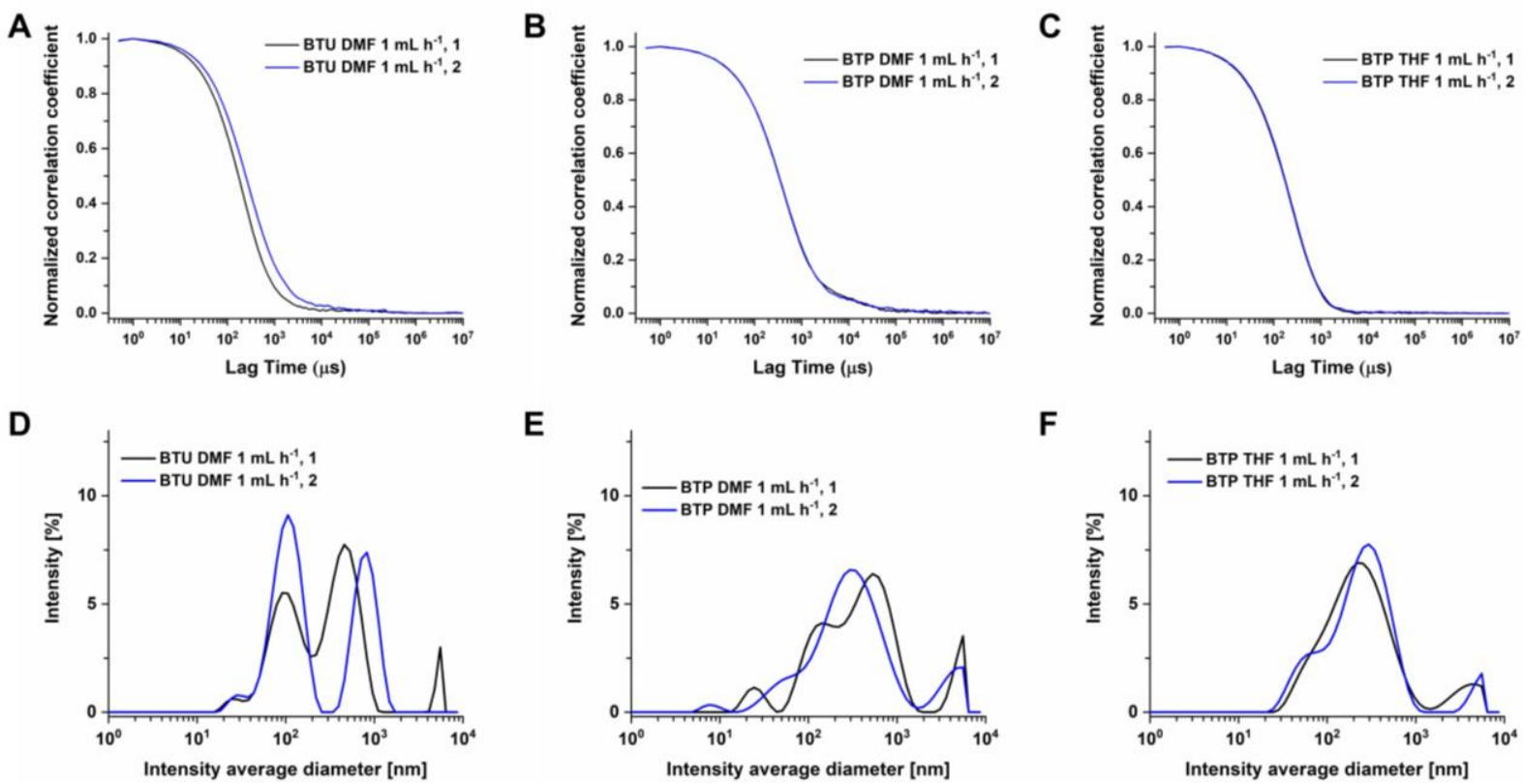

Figure S2: DLS correlograms showing the repeatability of the applied assembly procedure. A) B TU DMF 1 mL h ${ }^{-1}$, B) B TP DMF $1 \mathbf{~ m L ~ h} \mathbf{h}^{-1}$, and C) BTP THF $1 \mathbf{~ m L ~ h}^{-1}$. Each assembly procedure was performed twice, indicated by the numbers " 1 " and " 2 " in
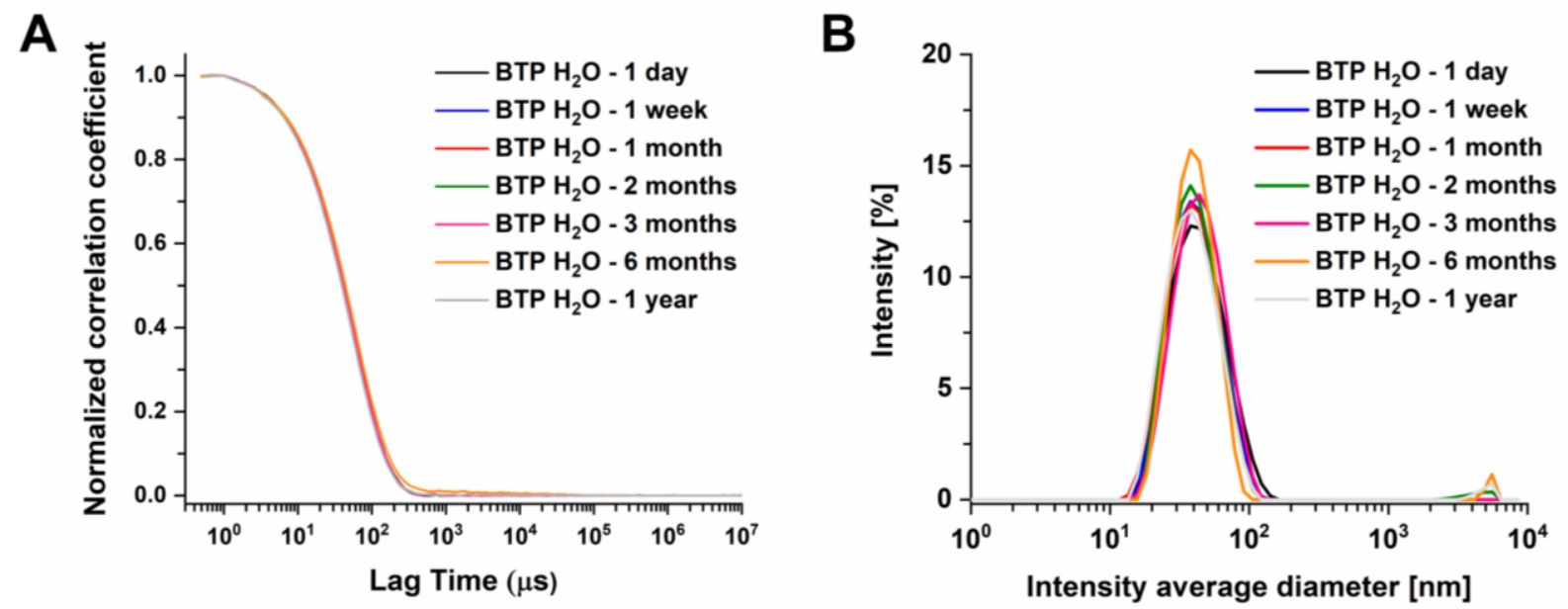

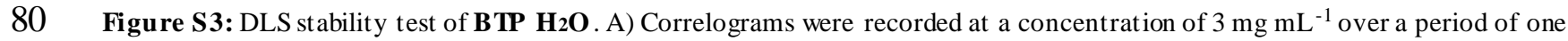
81 year. B) The corresponding intensity average diameters for the different time points. 


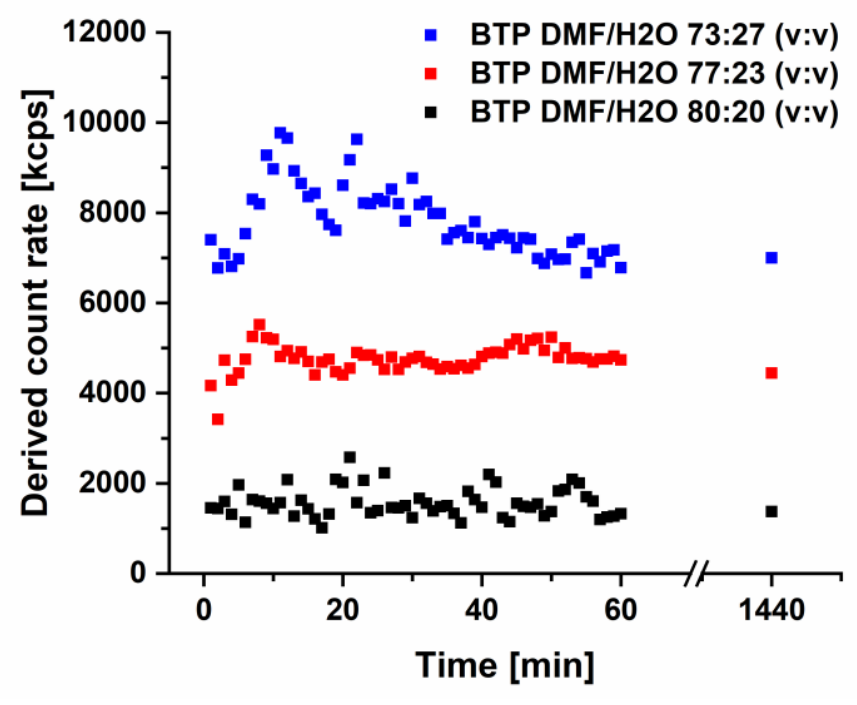

83 Figure S4: Time dependent evolution of derived count rates determined via DLS for BTP DMF at 20,23 and 27 v\% water.

84

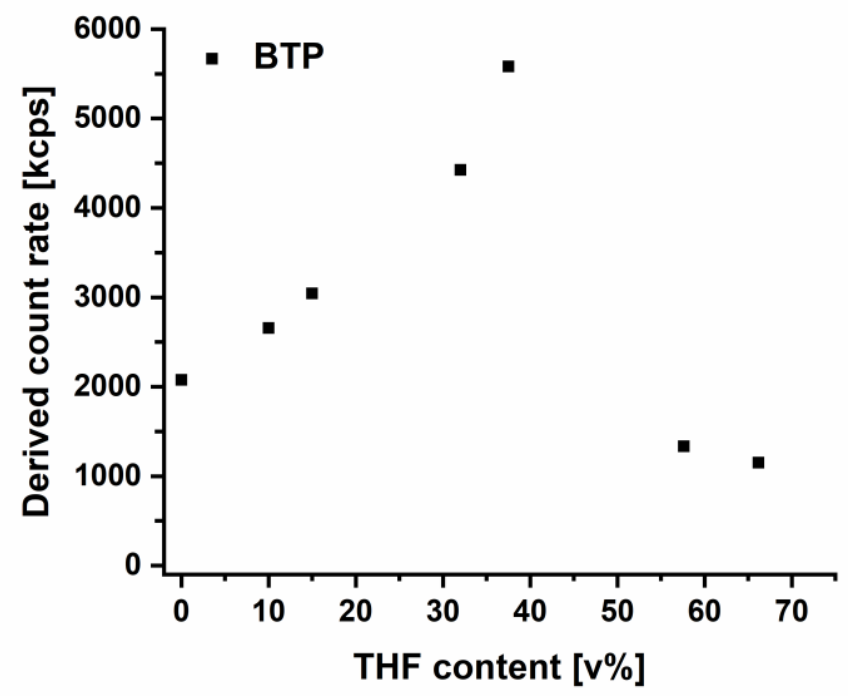


A

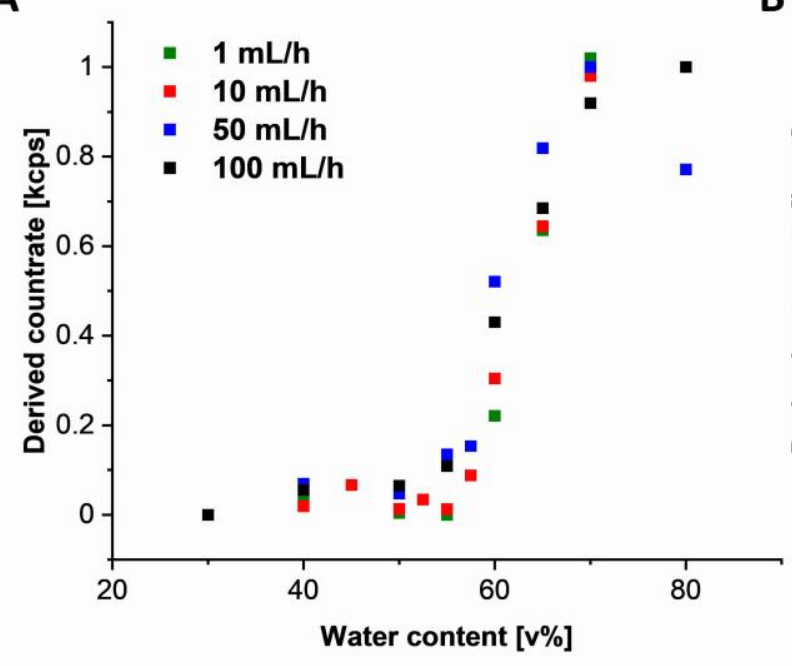

B

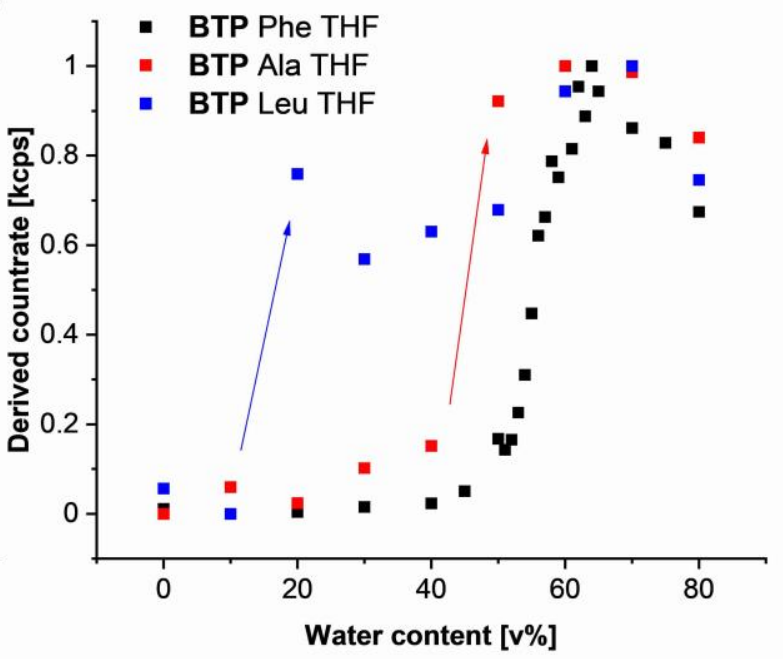

88 Figure S6: DLS measurement of the influence on the onset of aggregation for BTP in THF in dependence of the syringe pump

89 speed for the addition of water (A). Influence of the type of amino acid in BTP on the aggregation behavior upon increasing water

90 content in THF measured by DLS (B). The water was added via a syringe pump.

91

92

93 


\section{$94 \quad 2.2$ Cryo Transmission Electron Microscopy (cryo TEM)}

95 Samples were prepared on Ar plasma treated Quantifoil grids (R2/2). 8.5 $\mu \mathrm{L}$ of the solutions (3 mg

$96 \mathrm{~mL}^{-1}$ in $\mathrm{H}_{2} \mathrm{O}$ ) were applied onto the grids and vitrified in liquid ethane utilizing a FEI Vitrobot

97 Mark IV system (offset: $-3 \mathrm{~mm}$, blotting time: $1 \mathrm{~s}$ ). Samples were transferred into the cryo holder

98 (Gatan 626) utilizing the Gatan cryo stage, followed by transfer into the microscope keeping the

99 temperature below $-172{ }^{\circ} \mathrm{C}$ during the whole transfer and measurement process after vitrification.

100 Measurements were performed using a FEI Technai $\mathrm{G}^{2} 20$ operated at an acceleration voltage of

$101200 \mathrm{kV}$. Images were acquired with a Mega View (OSIS, Olympus Soft Imaging Systems) or an

102 Eagle 4k CCD camera. cryoTEM images in this study display only specific regions of interest that

103 are representative for the whole sample.

104

105 THF 1 mL h $\mathbf{h}^{-1}$ vs. THF que nch

106

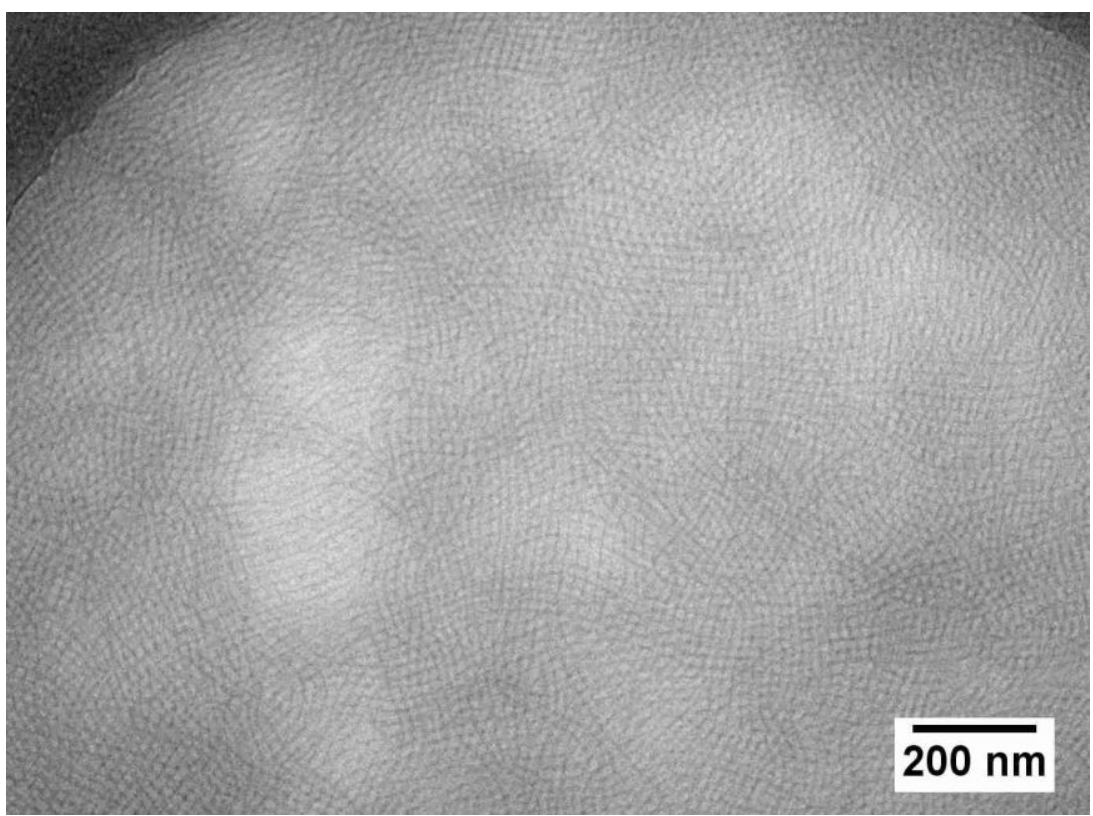




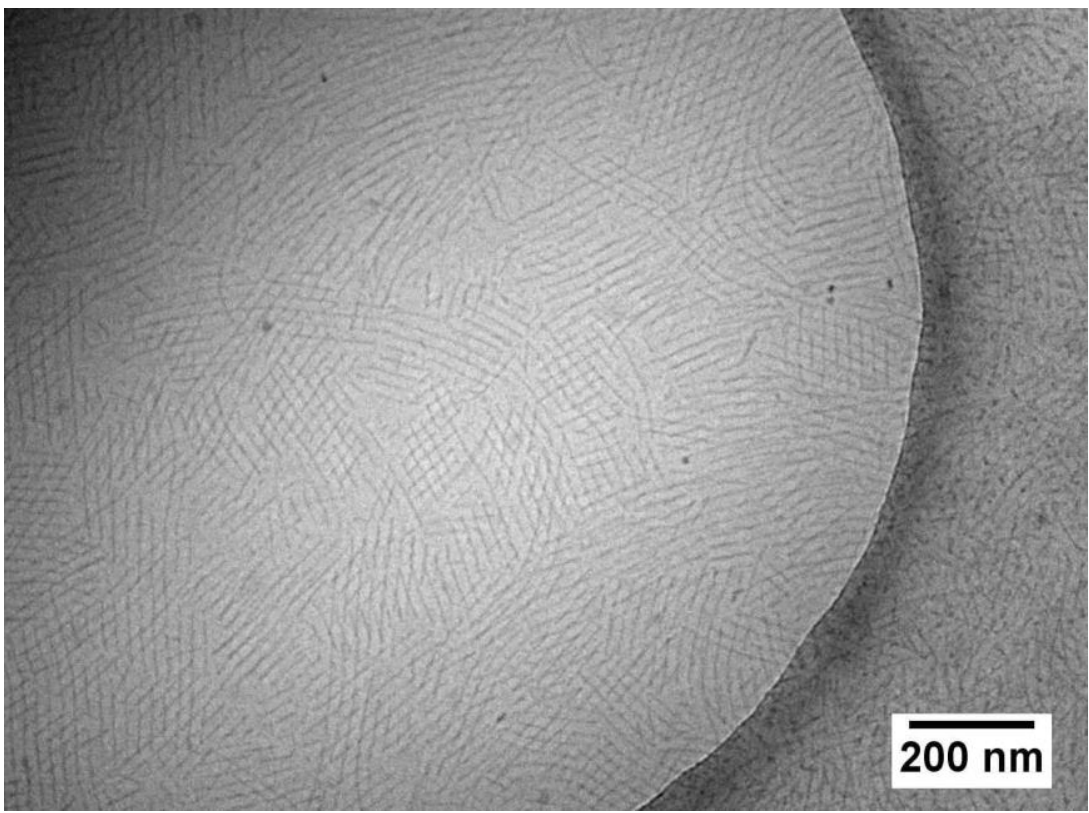

Figure S8: cryoTEM image of BTU THF quench in water $\left(c=1 \mathrm{mg} \mathrm{mL}^{-1}\right)$.

112

113

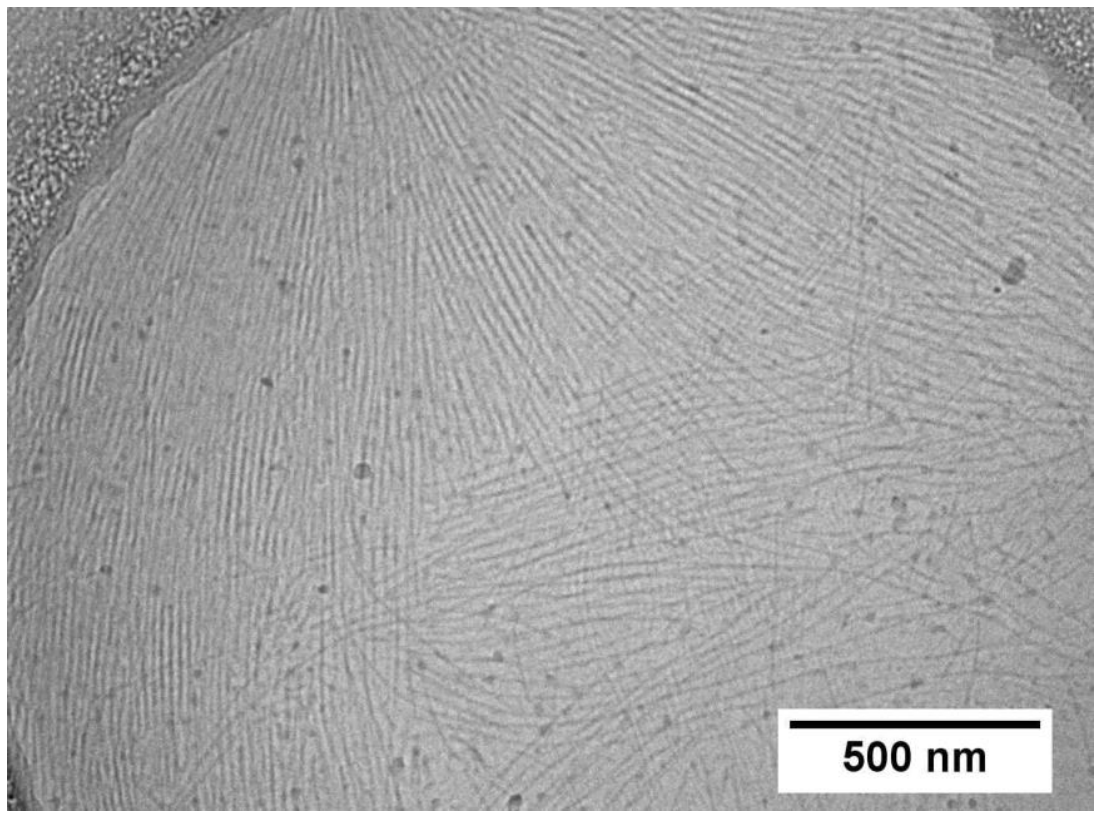

Figure S9: cryoTEM image of B TP THF $1 \mathbf{~ m L ~ h}^{-1}$ in water $\left(c=1 \mathrm{mg} \mathrm{mL}^{-1}\right)$. 

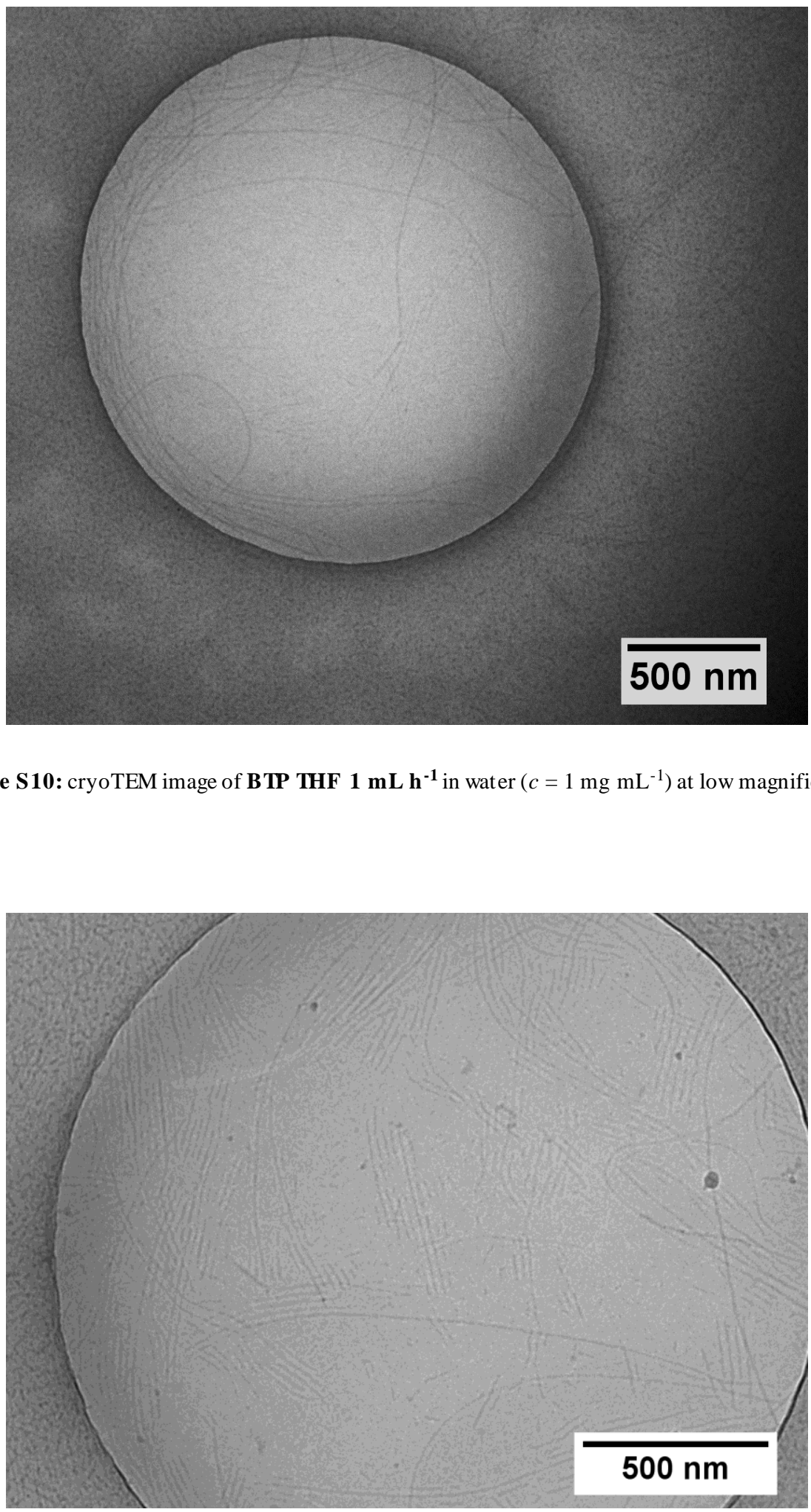

Figure S11: cryoTEM image of BTP THF $10 \mathbf{~ L ~ h ~}^{-1}$ in water $\left(c=1 \mathrm{mg} \mathrm{mL}^{-1}\right)$. 


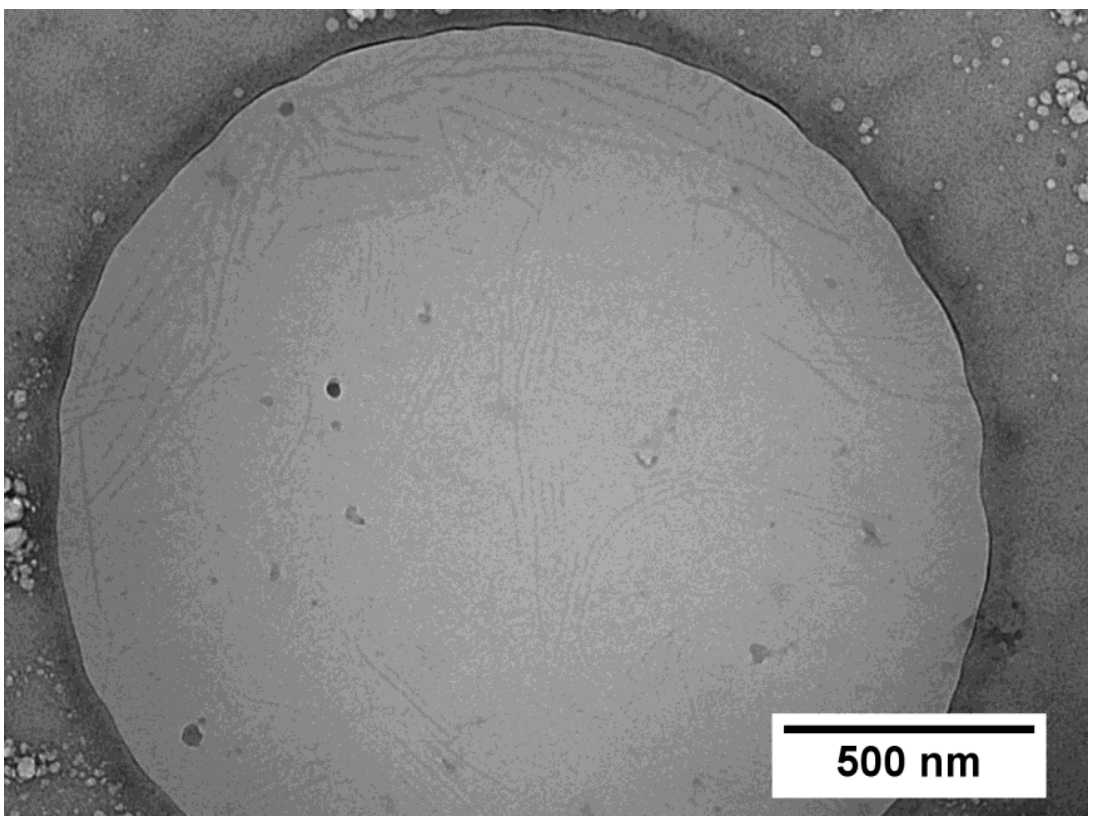

Figure S12: cryoTEM image of B TP THF $50 \mathrm{~mL} \mathrm{~h}^{-1}$ in water $\left(c=1 \mathrm{mg} \mathrm{mL}^{-1}\right)$

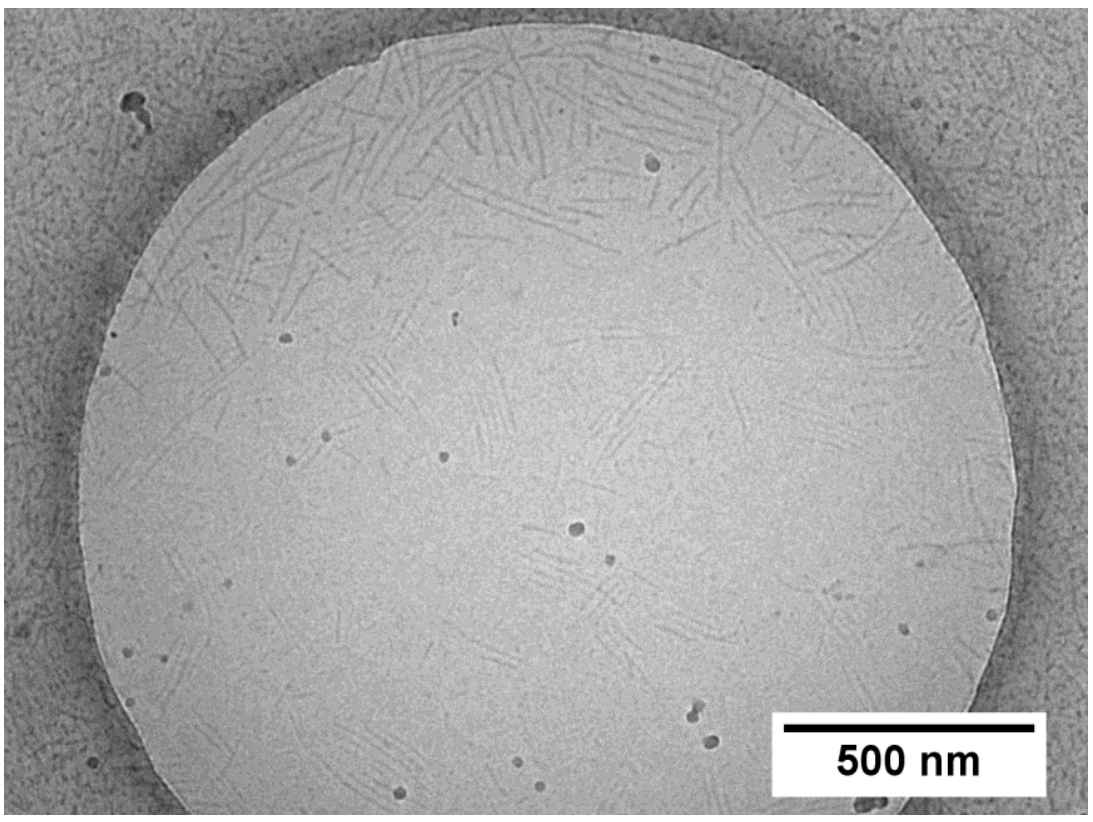

Figure S13: cryoTEM image of BTP THF $100 \mathbf{~ L ~ h}^{-1}$ in water $\left(c=1 \mathrm{mg} \mathrm{mL}^{-1}\right)$. 


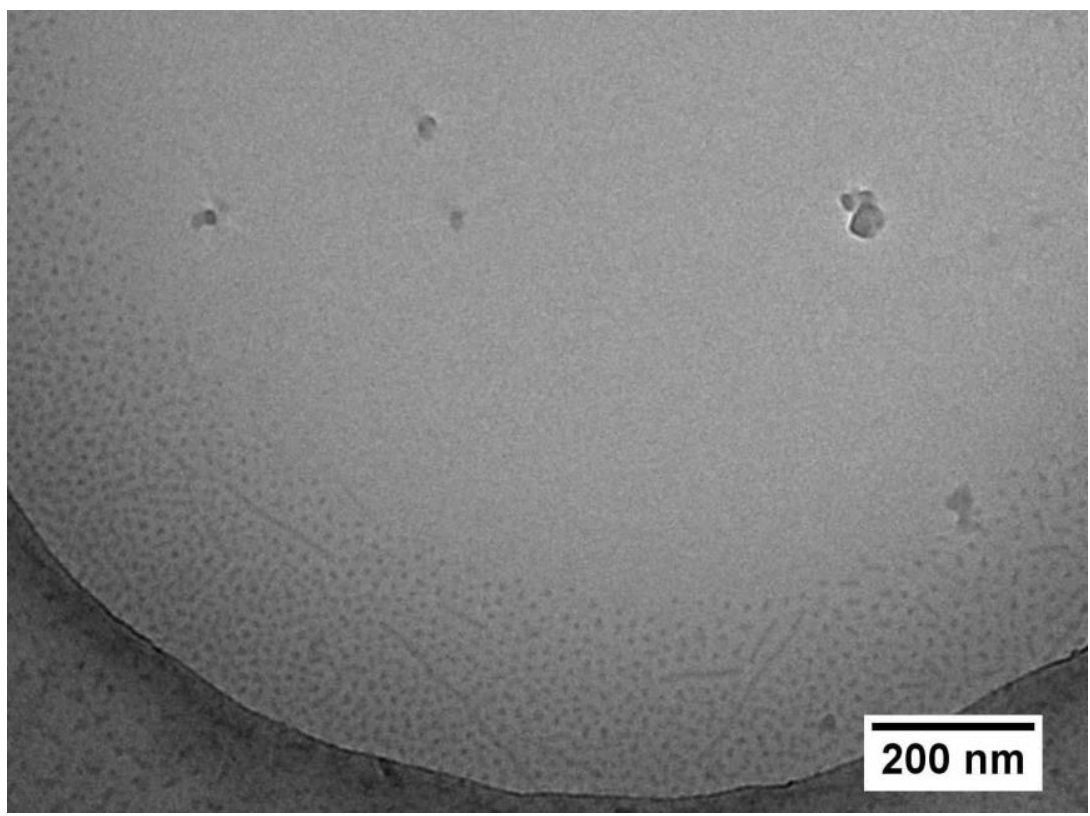

Figure S14: cryoTEM image of BTP THF quench in water $\left(c=1 \mathrm{mg} \mathrm{mL}^{-1}\right)$. 
A)

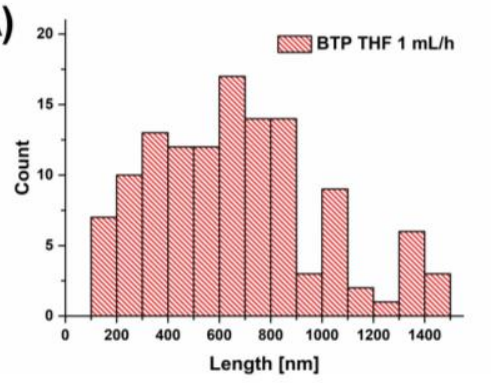

\begin{tabular}{|c|c|}
\hline Mean length & $666 \pm 332 \mathrm{~nm}$ \\
\hline Min. length & $102 \mathrm{~nm}$ \\
\hline Max. length & $1461 \mathrm{~nm}$ \\
\hline
\end{tabular}

D)

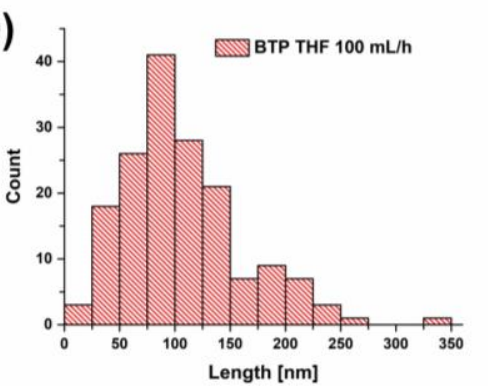

\begin{tabular}{|c|c|}
\hline Mean length & $107 \pm 54 \mathrm{~nm}$ \\
\hline Min. length & $14 \mathrm{~nm}$ \\
\hline Max. length & $333 \mathrm{~nm}$ \\
\hline
\end{tabular}

B)

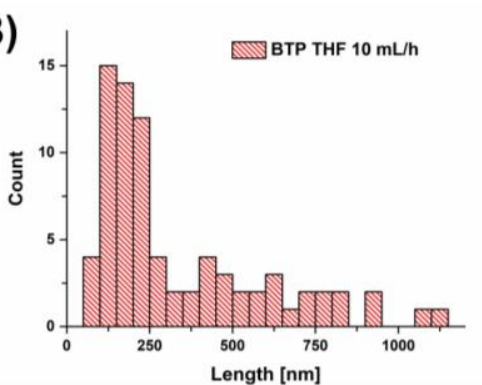

\begin{tabular}{|c|c|}
\hline Mean length & $356 \pm 296 \mathrm{~nm}$ \\
\hline Min. length & $67 \mathrm{~nm}$ \\
\hline Max. length & $1550 \mathrm{~nm}$ \\
\hline
\end{tabular}

E)

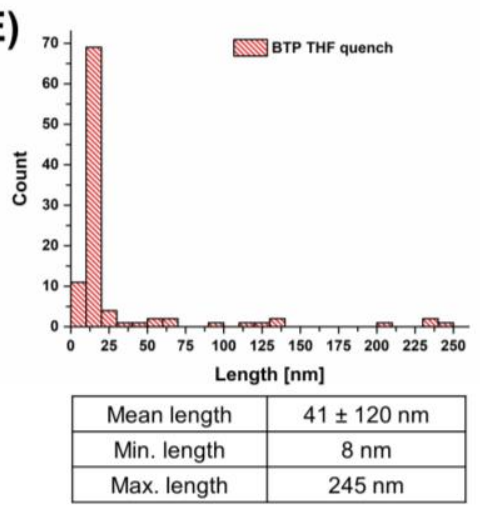

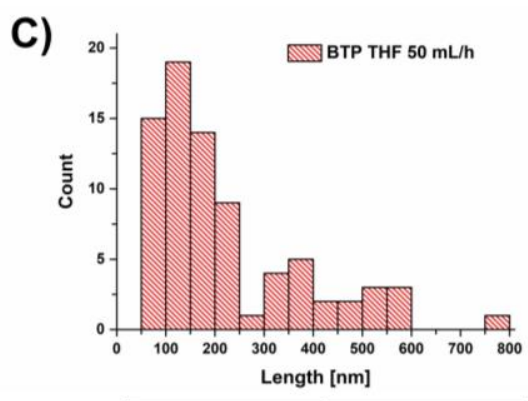

\begin{tabular}{|c|c|}
\hline Mean length & $224 \pm 153 \mathrm{~nm}$ \\
\hline Min. length & $51 \mathrm{~nm}$ \\
\hline Max. length & $767 \mathrm{~nm}$ \\
\hline
\end{tabular}

132 Figure S15: Histograms of fiber lengths obtained from cryoTEM images of BTP THF $1 \mathbf{m L ~ h}^{-1}$ (A), B TP THF $10 \mathbf{m L ~ h}^{-1}(B)$, BTP THF $50 \mathrm{mLh}^{-1}(\mathrm{C})$, BTP THF $100 \mathrm{mLh}^{-1}(\mathrm{D})$ and BTP quench (E).

134 


\section{$136 \quad 2.3$ Asymmetrical Flow Field-Flow Fractionation (AF4)}

137 AF4 measurements were performed on an AF2000 MT System from Postnova Analytics GmbH 138 Landsberg, Germany), equipped with a tip and focus pump (PN1130), an autosampler (PN5300), 139 and a channel oven unit (PN4020) set to $25^{\circ} \mathrm{C}$. The channel was coupled to a multiangle laser light 140 scattering (MALLS) detector (PN3621) equipped with a $532 \mathrm{~nm}$ laser and measuring 21 angles 141 (only $28^{\circ}$ to $148^{\circ}$ have been used for calculation of $R_{\mathrm{g}}$ and $M_{\mathrm{w}}$ ), a refractive index (RI) detector 142 (PN3150), and a UV-detector (PN3212) operating at a wavelength of $280 \mathrm{~nm}$. The channel had a 143 trapezoidal geometry with a nominal height of $350 \mu \mathrm{m}$. A regenerated cellulose (RC) membrane 144 from Postnova Analytics $\mathrm{GmbH}$ (10 kDa RC membrane) with a molar mass cutoff of $10 \mathrm{kDa}$ was 145 used as accumulation wall. As the mobile phase, an aqueous solution with $0.002 \mathrm{w} \%$ of $\mathrm{NaN}_{3}$ was 146 used. $50 \mu \mathrm{L}$ of the sample at a concentration of $1 \mathrm{mg} \mathrm{mL}^{-1}$ was injected with an injection flow rate

147 of $0.2 \mathrm{~mL} \mathrm{~min}^{-1}$, a focus flow rate of $0.8 \mathrm{~mL} \mathrm{~min}^{-1}$, and a cross-flow rate of $0.7 \mathrm{~mL} \mathrm{~min}-1$, resulting 148 in a detector flow rate of $0.3 \mathrm{~mL} \mathrm{~min}^{-1}$. The focusing time was 4 min before switching to elution 149 at an exponentially decaying crossflow from $0.7 \mathrm{~mL} \mathrm{~min}^{-1}$ to $0.2 \mathrm{~mL} \mathrm{~min}{ }^{-1}$ within $76.2 \mathrm{~min}$. 150 Thereafter, the crossflow profile was set to decay in a linear way from $0.05 \mathrm{~mL} \mathrm{~min}^{-1}$ to $1510.04 \mathrm{~mL} \mathrm{~min}^{-1}$ within $71 \mathrm{~min}$ (Figure S21). Before the start of the next measurement, a rinsing step 152 was performed at $1.5 \mathrm{~mL} \mathrm{~min}^{-1}$ flow of the tip pump only for $20 \mathrm{~min}$. After each sample 153 measurement, a blank measurement was run, which was subtracted from the data of the sample 154 measurement for analysis. The RI-detector was used as the concentration-sensitive detector $155\left(\mathrm{dn} / \mathrm{dc}_{\mathrm{BTU}} 1.47 \mathrm{~mL} / \mathrm{g}\right.$ and $\left.\mathrm{dn} / \mathrm{dc}_{\mathrm{BTP}} 1.48 \mathrm{~mL} / \mathrm{g}\right)$ and the MALLS data was analysed via a ZIMM plot 156 analysis to obtain the radii of gyration $\left(R_{\mathrm{g}}\right)$ and the molar mass at the specified elution times. 


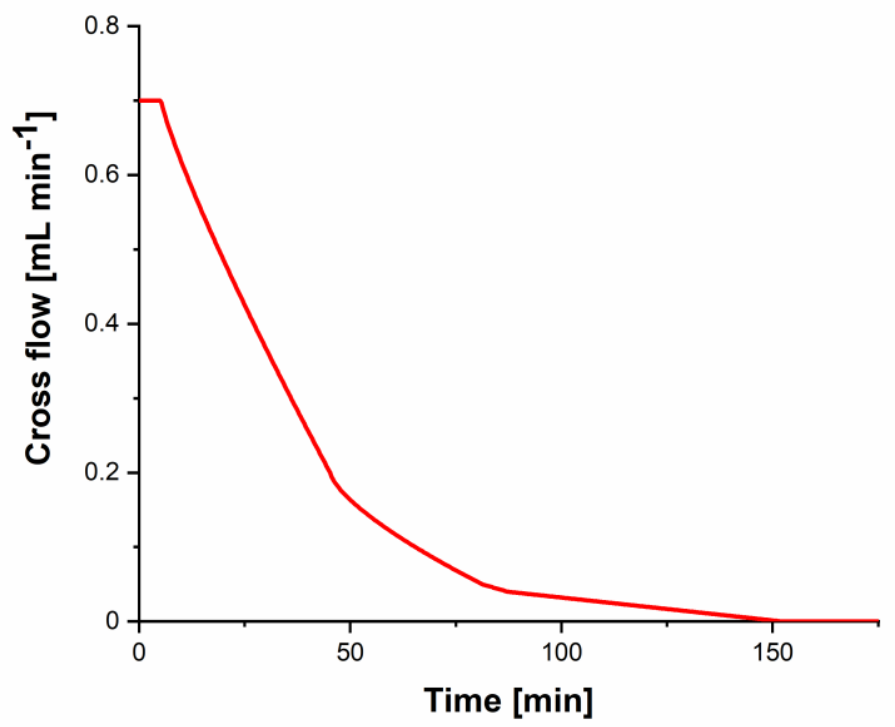

158

Figure S16: Cross-flow profile applied for all AF4-MALLS measurements in this manuscript.

A

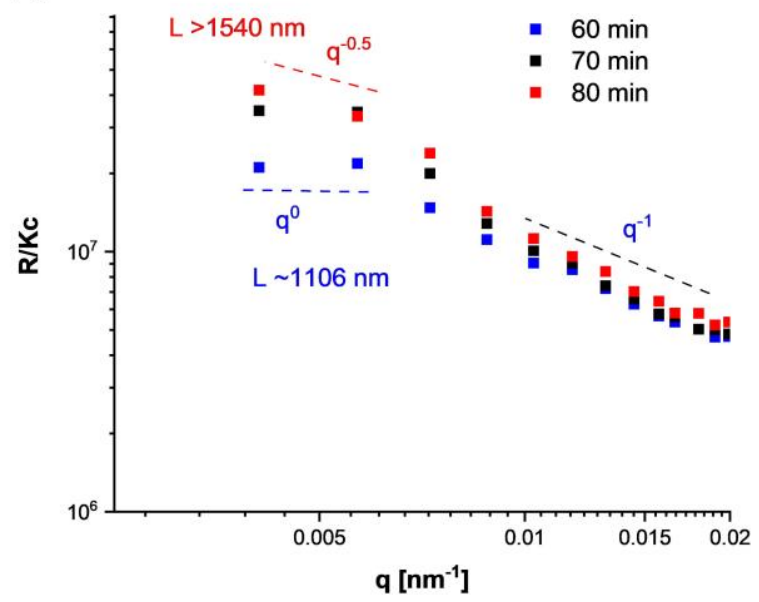

B

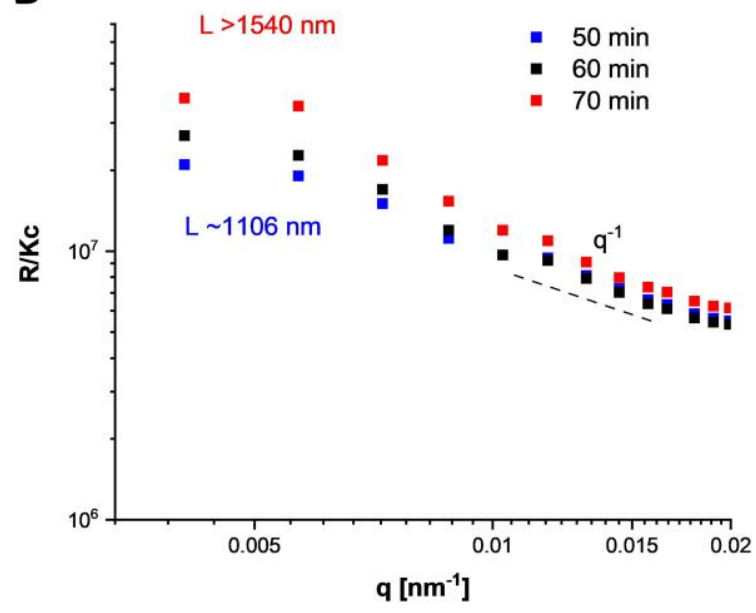

161 Figure S17: Scattering intensity plotted against the scattering vector $q$ for B TU THF $\mathbf{1} \mathbf{~ m L ~ h}^{\mathbf{- 1}}(\mathrm{A})$ and $\mathbf{B}$ TP $\mathbf{T H F} \mathbf{1} \mathbf{~ m L ~ h} \mathbf{h}^{\mathbf{- 1}}(\mathrm{B})$

162 and the maximum observable length derived from the change of the $q^{-1}$ dependency to a $q^{0}$ plateau at low scattering values. 

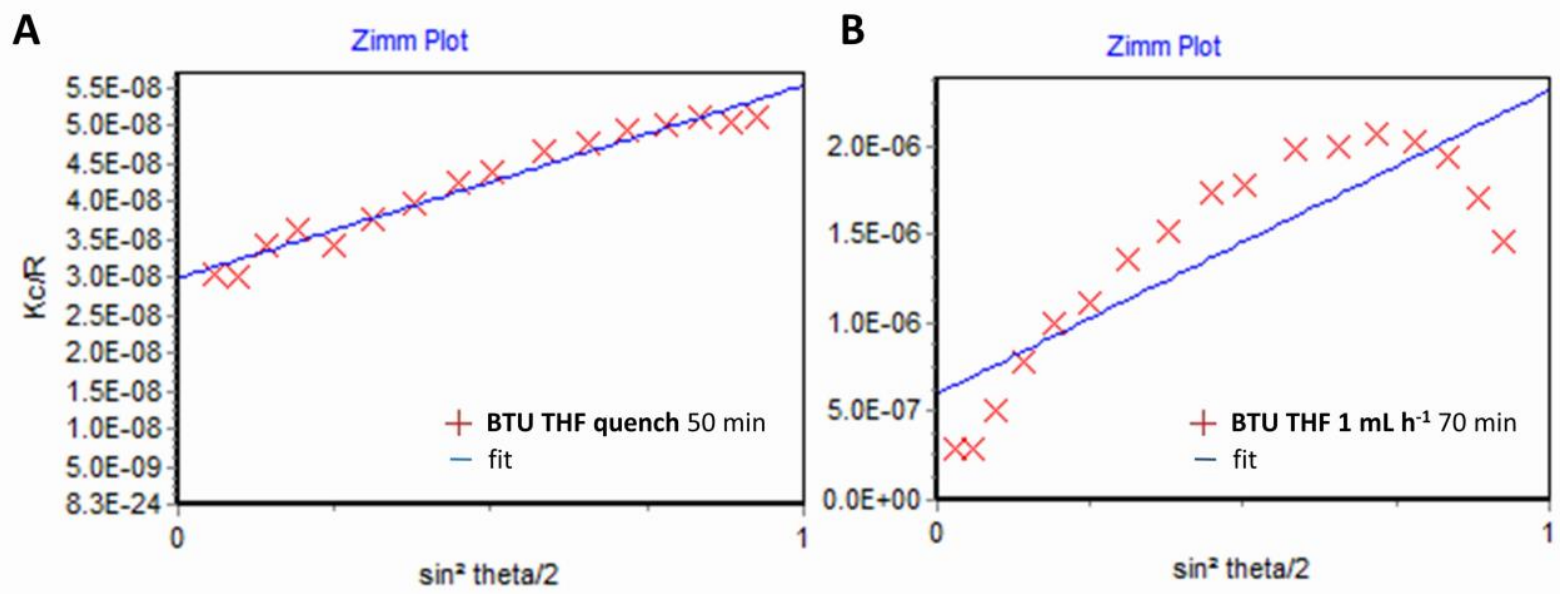

163

164 Figure S18: Zimm plots for BTU THF quench at 50 min resulting in an $R_{\mathrm{g}}$ of $80 \mathrm{~nm}$ (A) and for BTU THF $\mathbf{1} \mathbf{~ m L ~ h}^{\mathbf{- 1}}$ at $70 \mathrm{~min}$ 165 where Zimm fitting is not accurate anymore (B). 
A

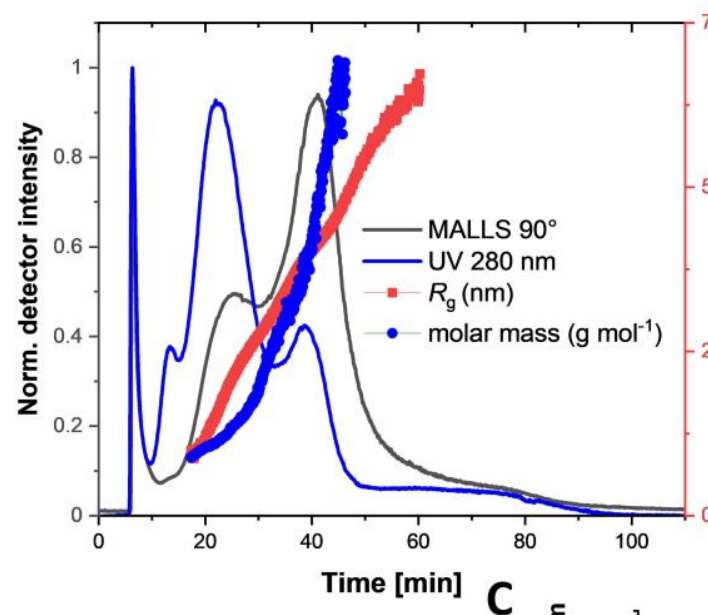

B

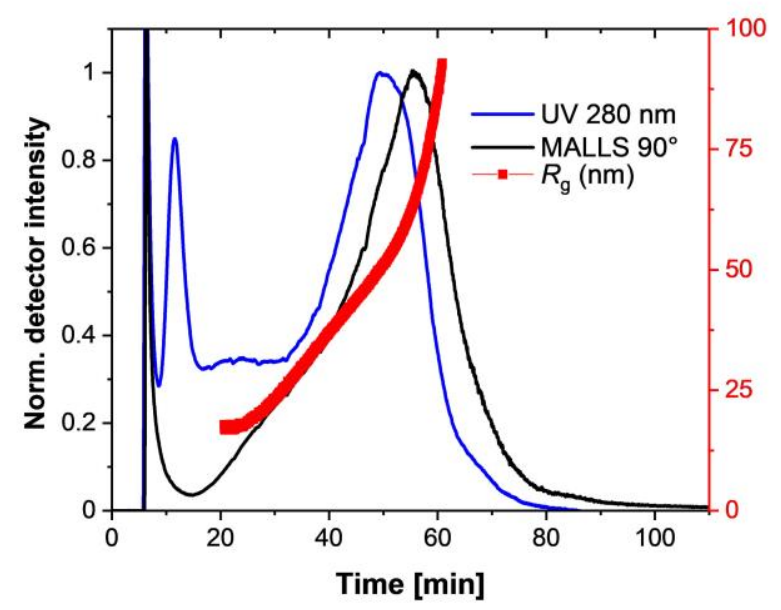

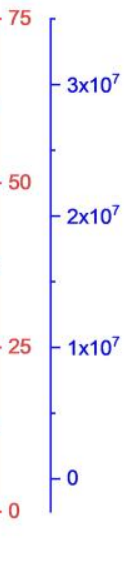

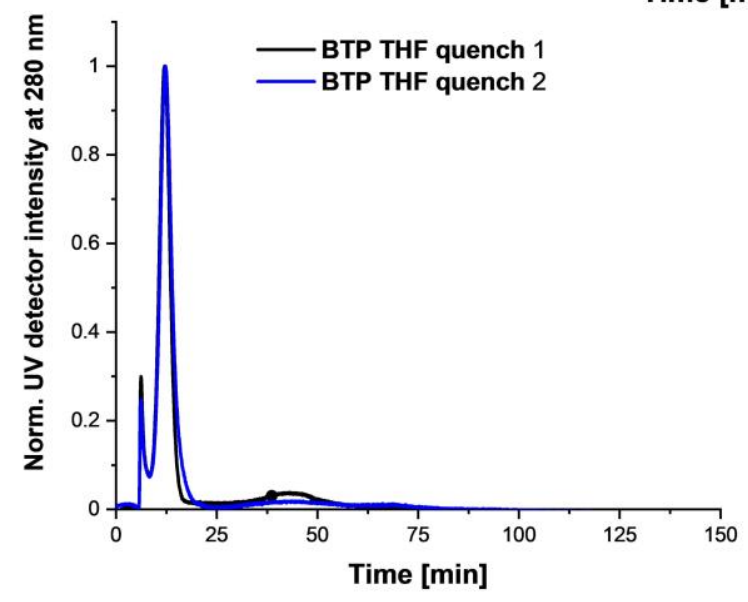

Figure S19: MALLS 90 (black), UV trace (blue), Rg (red) and molar mass (blue) obtained by Zimm analysis for BTU THF quench 1 (A) and BTU THF quench 2 (C). cryoTEM image of BTU THF quench $1\left(c=1 \mathrm{mg} \mathrm{mL}^{-1}\right)$. B TP THF quench 1 and 2

170 (D). 
A

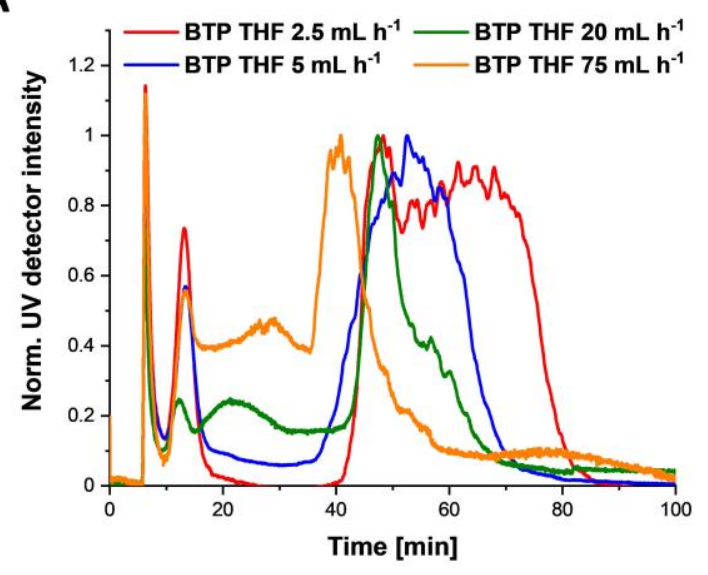

B

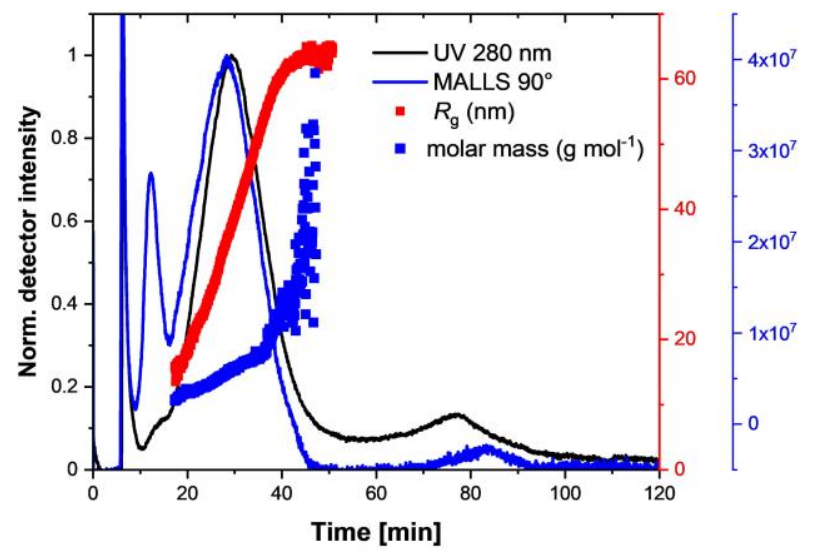

C

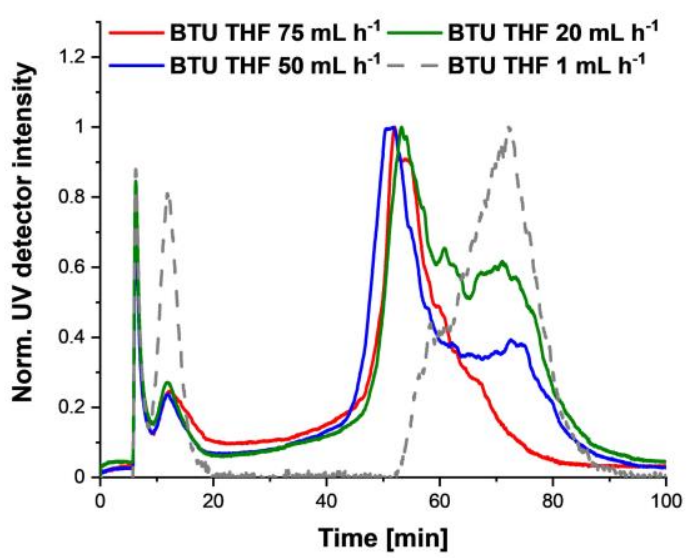

173 Figure S20: Impact of the water addition rate on the resulting B TP THF assemblies (A) and BTU THF assemblies (C) (elution profile monitored via UV detector). MALLS $90^{\circ}$ (black), UV trace (blue) and $\mathrm{R}_{\mathrm{g}}$ (red) obtained via Zimm plot for BTP THF $100 \mathrm{~mL} \mathrm{~h}^{-1}(\mathrm{~B})$. 

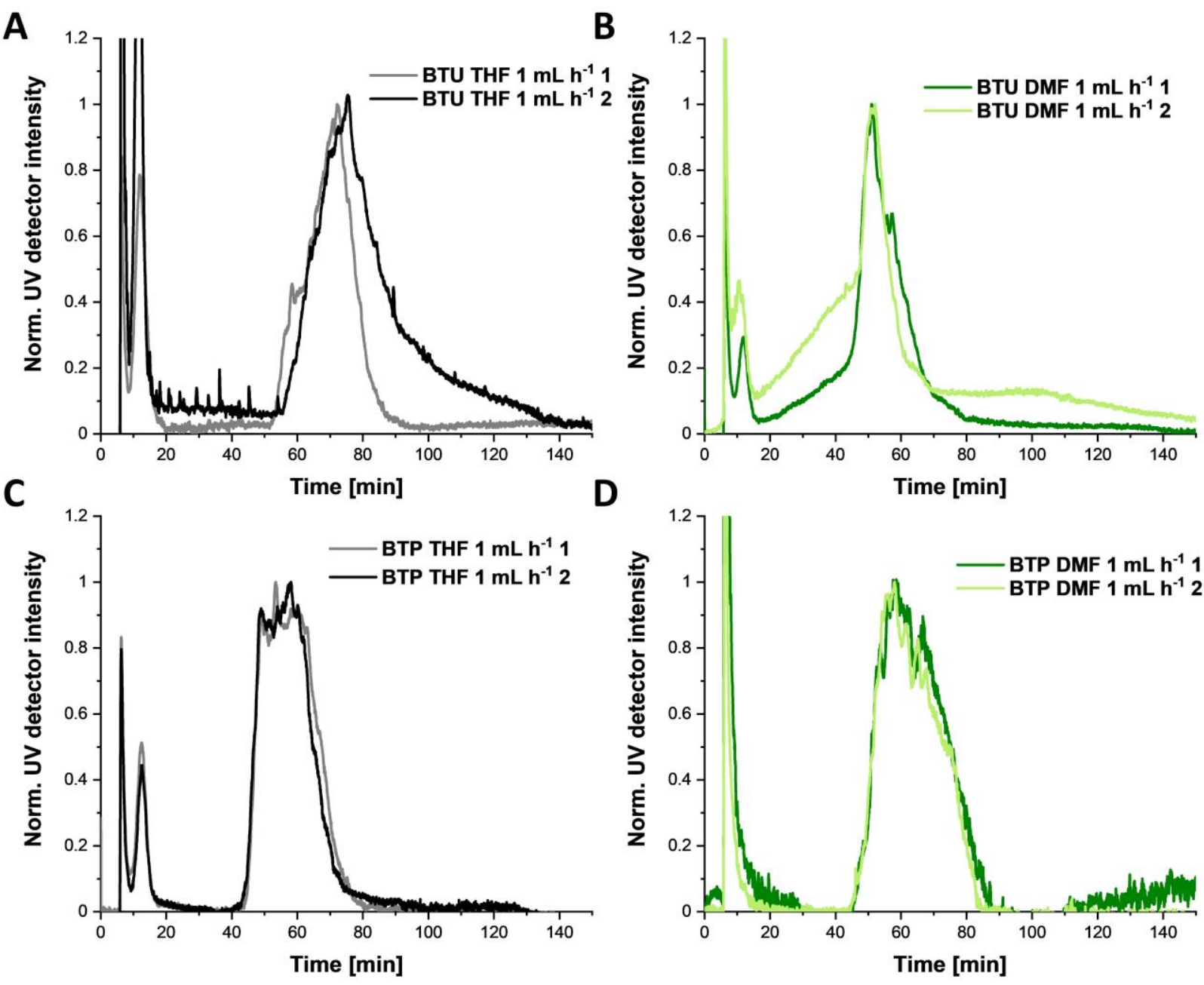

178 Figure S21: Repeatability of the solvent switch procedure shown for B TU THF $1 \mathbf{~ m L ~ h}^{-1}$ (A), B TU DMF 1 mL h ${ }^{-1}$ (B), B TP THF $1 \mathrm{~mL} \mathrm{~h}^{-1}(\mathrm{C})$ and $B$ TP DMF $1 \mathrm{~mL} \mathrm{~h}^{-1}$ (D). 
A

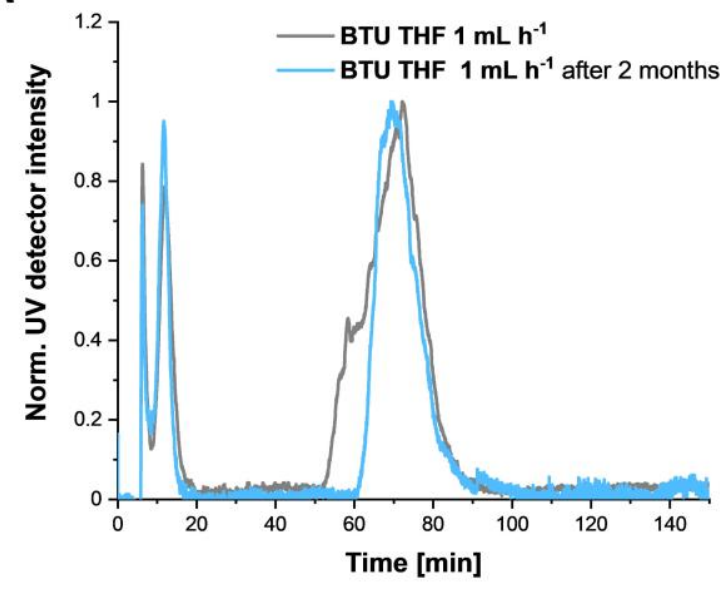

B

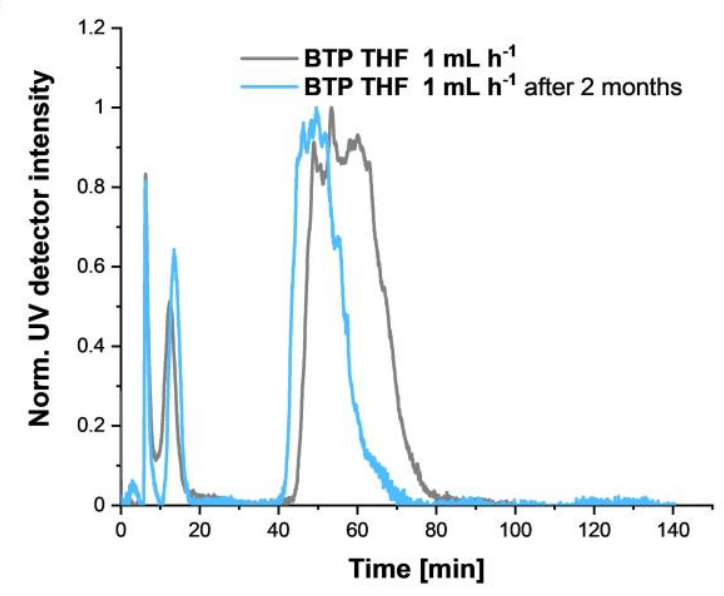

182 Figure S22: Normalized UV traces of BTU THF $1 \mathbf{~ m L ~ h}^{-1}$ (A) and BTP THF $1 \mathbf{~ m L ~ h}^{-1}$ (B) (grey) and after 2 months (blue). Slight 183 deviations result from variations in the membranes and pressure differences in the AF4 system.

184

A

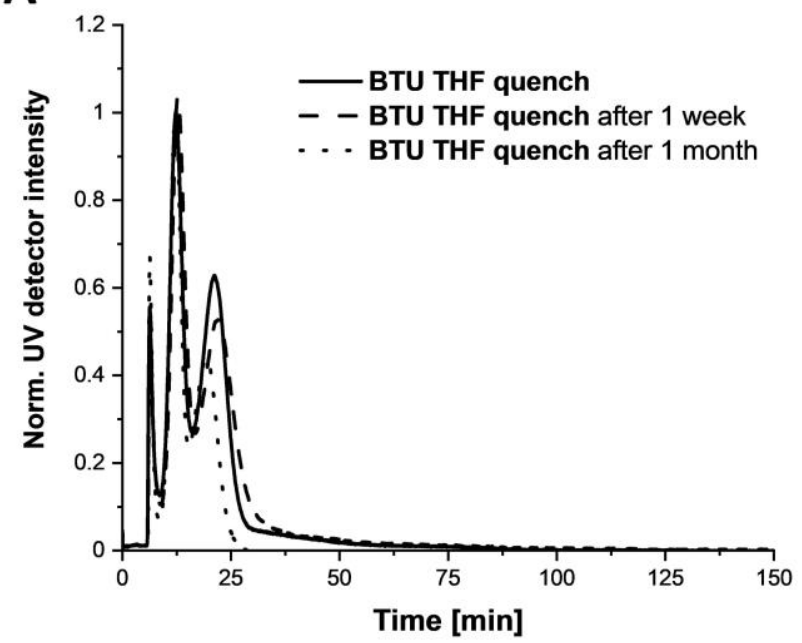

B

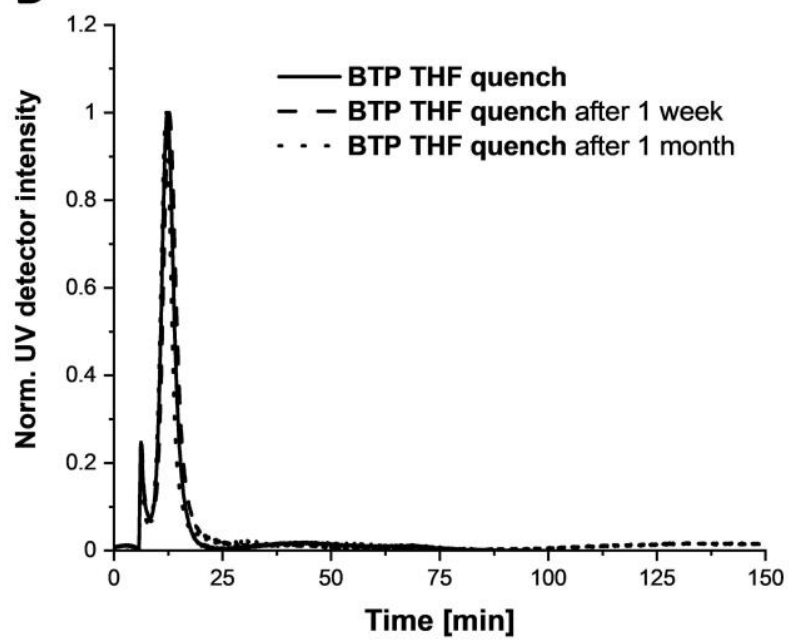




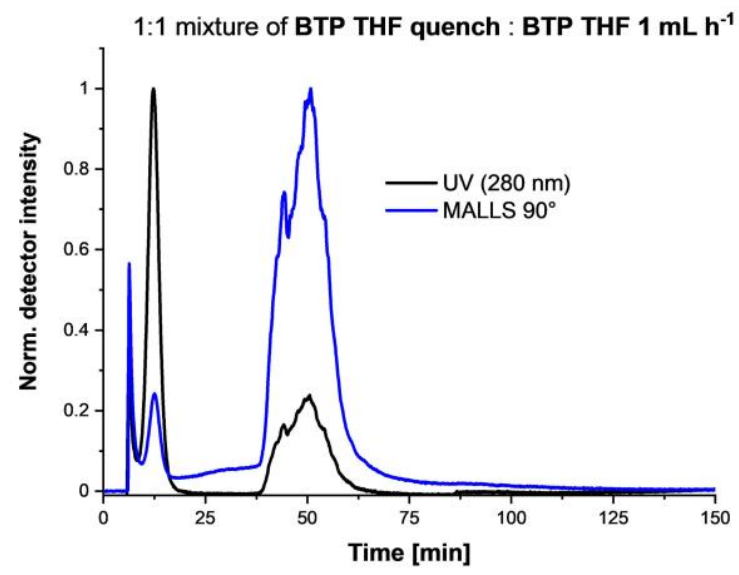

189 Figure S24: AF4 elution profile proving the stability of a 1:1 (v/v) mixture of BTP THF 1 mL h $\mathbf{h}^{-1}$ and B TU THF quench (B) 190 measured after several days (B).

\subsubsection{Seeded-growth experiments:}

193 For the seeded growth experiment at high THF contents, 0.5-1 eq of monomer in a $\mathrm{THF} / \mathrm{H}_{2} \mathrm{O}$ 194 solvent mixture (1:1 v:v, $5 \mathrm{mg} \mathrm{mL}^{-1}$ for BTP, pure THF for BTU, below the aggregation point) 195 were added via pipette in small drops under vigorous shaking to a solution of B TU or BTP quench $196(1 \mathrm{mg} / \mathrm{mL})$ at $30 \mathrm{v} \%$ THF in water. The THF was evaporated and the sample was measured with 197 the herein used AF4 setup. To check the influence of the dynamics at different THF contents, 198 additionally to the high (slightly above the aggregation point) THF contents, additional monomer 199 was added at low (below the aggregation point) THF contents. It can be seen that at THF contents

200 below the aggregation point no elongation of the existing particles can be observed but an 201 additional quenching into small structures. At THF contents higher than the initial THF content 202 were aggregation takes place, a growth of the structures can be observed. However, this growth is 203 not only a controlled elongation at active ends of the fibers but also a competing aggregation 204 resulting in large and very disperse distributions. 


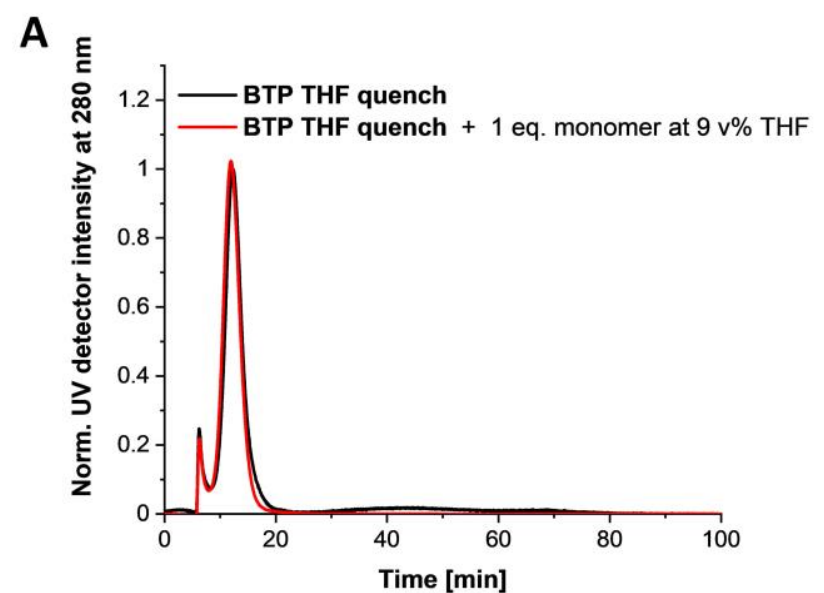

B

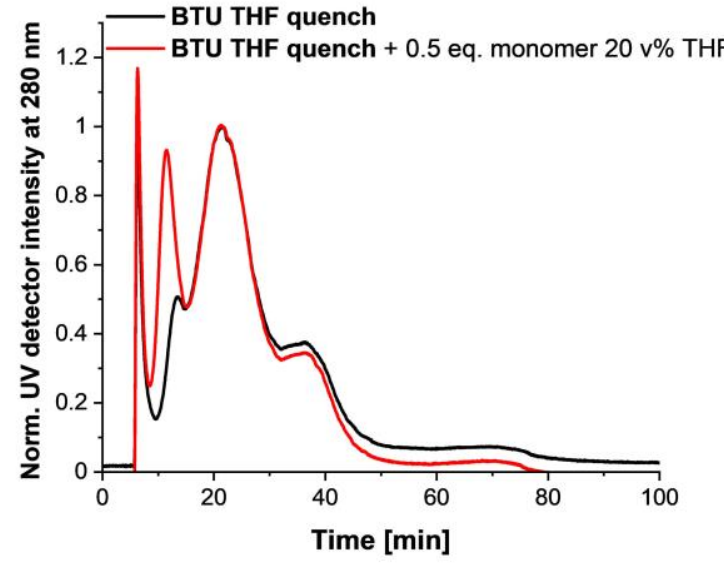

C

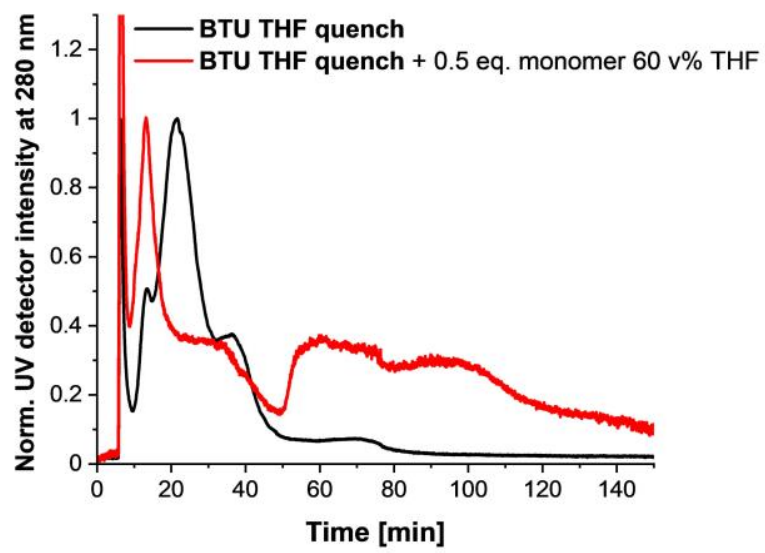

Figure S 25: AF4 elution profile of B TP quench before (black) and after the addition of 1 eq monomer in THF $(9 \mathrm{v} \%$ THF in the solution in the end (A) and of BTU quench before (black) and after the addition of 0.5 eq monomer in THF ( $20 \mathrm{v} \% \mathrm{THF}$ in the solution in the end (B) and $60 \mathrm{v} \%$ of THF (C). (THF was removed before the measurement by evaporation). 


\section{$210 \quad$ 2.4 Circular dichroism (CD)}

211 CD spectra were recorded on a JASCO J-820KS spectrophotometer. Each sample was measured 212 five times at a concentration of $1.5 \mathrm{mg} \mathrm{mL}-1$ in water using a quartz cell with a path length of 1 $213 \mathrm{~mm}$.

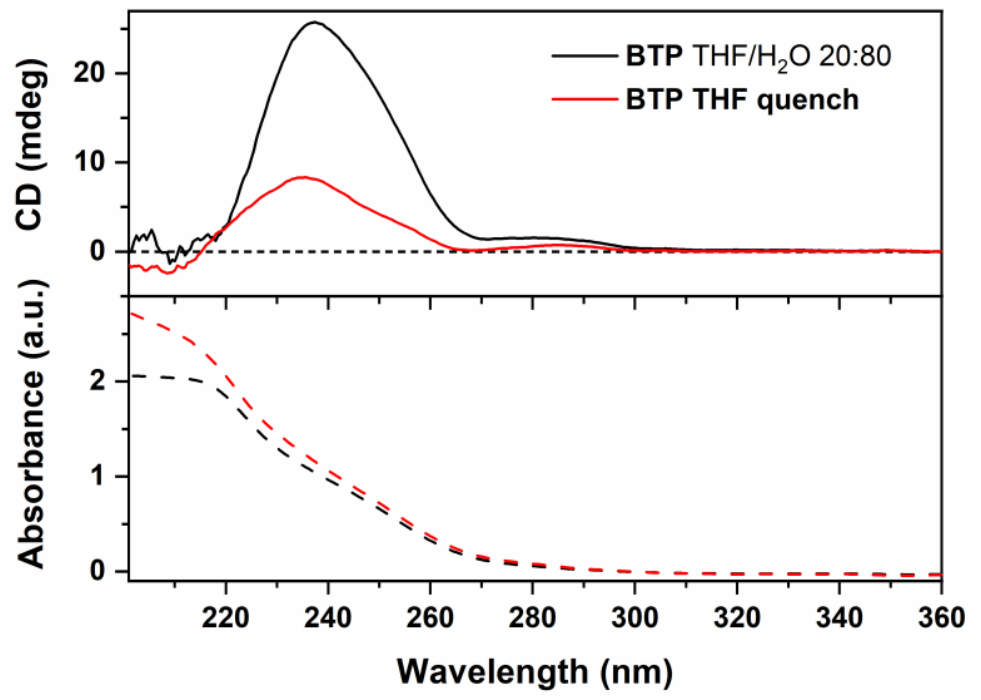

215 Figure S 26: CD spectra of BTP THF quench in pure $\mathrm{H}_{2} \mathrm{O}$ (red) and BTP in a 20:80 (v:v) THF: $\mathrm{H}_{2} \mathrm{O}$ mixture (black) for 216 comparison. Both curves a characteristic for right-handed helical structures, however, for B TP THF quench the intensity is 217 decreased compared to B TP 20:80, which can be ascribed to a lower overall aggregation. 
220 FT-IR spectra were measured with an ATR unit at a Bruker Invenio S.

A

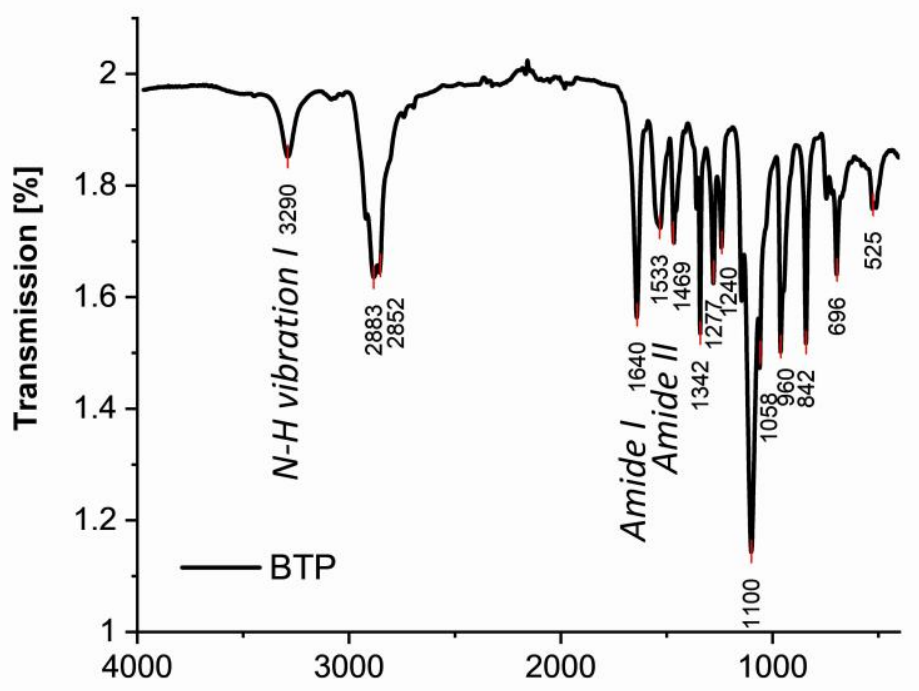

B

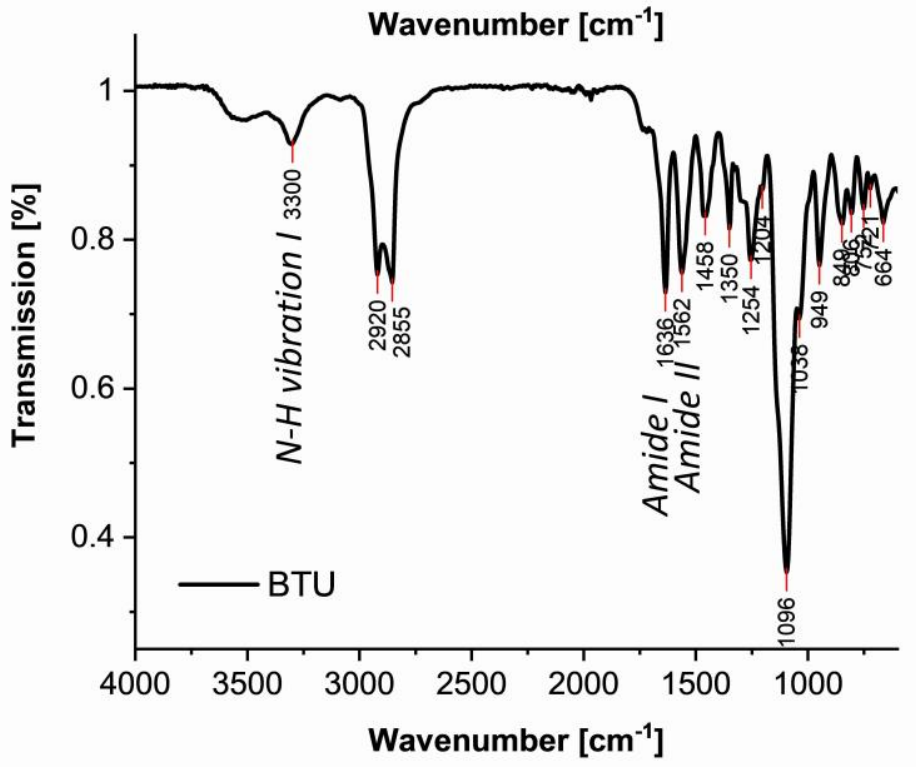

Figure S 27: FT-IR spectra of BTP (A) and BTU (B) measured on ATR unit. 


\section{$224 \quad 2.6$ Nuclear magnetic resonance (NMR) spectroscopy}

$225{ }^{1} \mathrm{H}-\mathrm{NMR}$ spectra were measured with a Bruker spectrometer (300 MHz) equipped with an Avance 226 I console, a dual $1 \mathrm{H}$ and 13C sample head and a 120x BACS automatic sample changer. The

227 chemical shifts of the peaks were determined by using the residual solvent signal as reference and 228 are given in ppm in comparison to TMS.

229

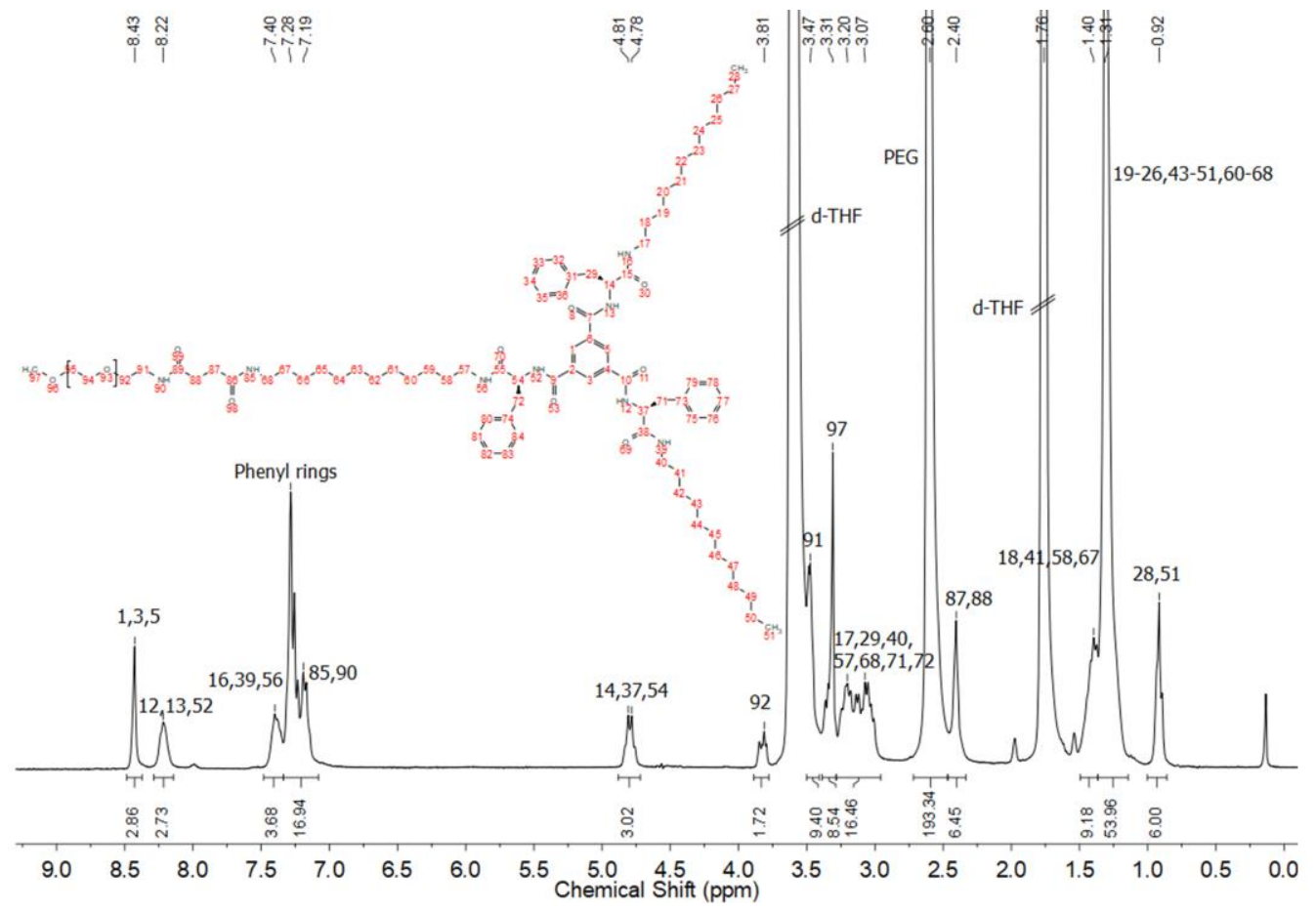

231 Figure $\mathbf{S} 28:{ }^{1} \mathrm{H}-\mathrm{NMR}$ of $\mathbf{B}$ TP in $\mathrm{d}_{8}$-THF. 


\section{$233 \quad 2.7$ Small angle $x$-ray scattering}

234 The SAXS measurements in $\mathrm{DMF} / \mathrm{H}_{2} \mathrm{O}$ solvent mixtures were performed at BL-40B2 at SPring-8, 235 Hyogo Prefecture, Japan. The distances between the sample and detector were $4 \mathrm{~m}$. The wavelength 236 was $0.1 \mathrm{~nm}$. The sample was prepared at a starting concentration of $10 \mathrm{mg} \mathrm{mL}^{-1}$ and diluted 237 successively to $0.3 \mathrm{mg} \mathrm{mL}^{-1}$ by addition of water.

238 The data was fit by a solid cylinder with a radius of Gaussian distribution.

239 Table S 1 Fit parameters for a solid cylinder (the scattering length density was calculated to be $0.001018 \mathrm{~nm}$ and the length was 240 fixed to $200 \mathrm{~nm}$ since the fiber length can not be extracted from SAXS data but should exceed approx. $150 \mathrm{~nm}$ ).

\begin{tabular}{l|lll} 
Water content $(v \%)$ & $N$ & $R_{c y l}(\mathrm{~nm})$ & $S D(\mathrm{~nm})$ \\
\hline 23 & 0.00033 & 5.07 & 1.06 \\
36 & 0.00038 & 5.93 & 1.07 \\
46 & 0.00036 & 6.24 & 1.11 \\
57 & 0.00039 & 6.08 & 1.51 \\
67 & 0.00047 & 6.36 & 1.27 \\
76 & 0.00039 & 6.20 & 1.42 \\
83 & 0.00057 & 6.08 & 1.31 \\
100 & 0.00025 & 6.28 & 1.34
\end{tabular}

243 Simultaneously, for the fitting of the fibrillar SAXS profiles, a model of a core-shell cylinder was 244 used, described by the following expression:

$$
I(q)=\frac{L \pi}{q} *\left\{2 \pi R_{\mathrm{c}}^{2}\left(\rho_{\mathrm{c}}-\rho_{\mathrm{S}}\right) \frac{J_{1}\left(q R_{\mathrm{c}}\right)}{q R_{\mathrm{c}}}+2 \pi R_{\mathrm{S}}^{2} \rho_{\mathrm{s}} \frac{J_{1}\left(q R_{\mathrm{S}}\right)}{q R_{\mathrm{S}}}\right\}^{2}
$$

246 This yielded also adequate fits for the SAXS data and the similar constant trend upon increasing 247 water addition in the radius can be observed. 


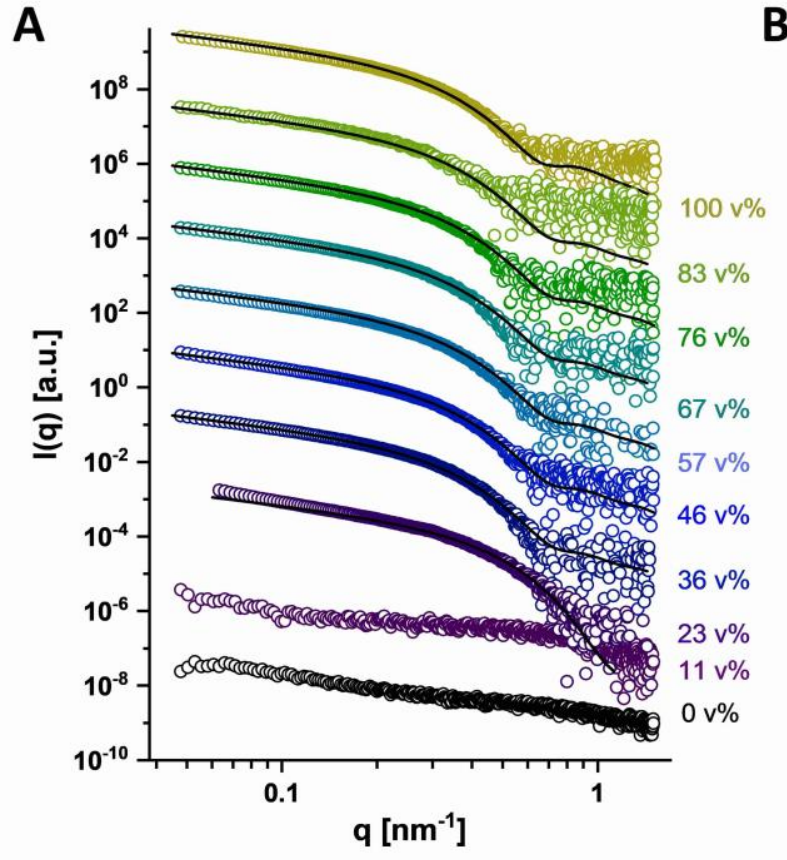

B

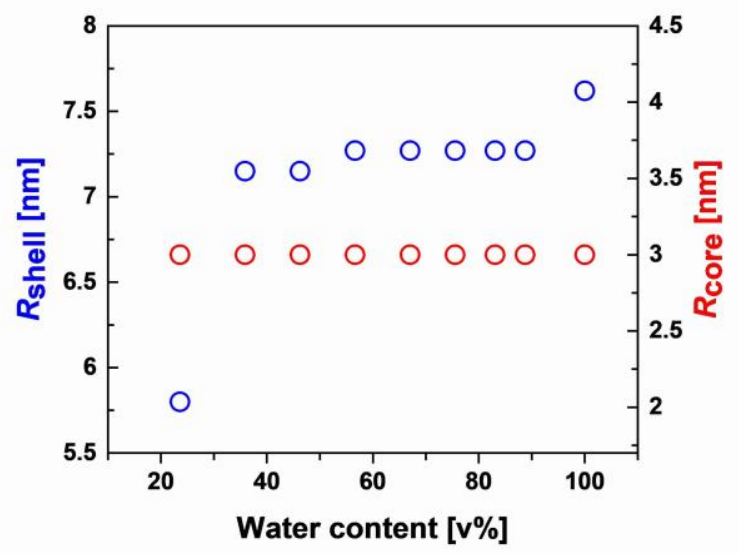

250 Figure S 29: Scattering profile of B TP in DMF at different water contents and the respective core-shell cylindrical fits (A).

251 Evolution of the core (red) and shell (blue) radius of the cylinder with increasing water content (B).

252 Table $\mathbf{S} 2$ Fit parameters for a core-shell cylinder where $R_{c}$ was estimated to be $3 \mathrm{~nm}$ and $\mathrm{R}_{\mathrm{s}}$ was fitted accordingly. The resulting 253 calculations were proven by checking the fitted $\mathrm{R}_{\mathrm{g}_{-}}$fitted $v$. the $\mathrm{R}_{\mathrm{g} \_ \text {experiement }}$ calculated from the measured data via Guinier plot .

\begin{tabular}{l|llllll}
$\begin{array}{l}\text { Water content } \\
(v \%)\end{array}$ & $R_{c}(\mathrm{~nm})$ & $R_{s}(\mathrm{~nm})$ & $\rho_{c}\left(\mathrm{e} \mathrm{nm}^{-3}\right)$ & $\rho_{s}\left(\mathrm{e} \mathrm{nm}^{-3}\right)$ & $R_{\text {gc_fitted }}(\mathrm{nm})$ & $\begin{array}{l}R_{\text {g__experiment }} \\
(\mathrm{nm})\end{array}$ \\
\hline 23 & 3 & 5.8 & 307 & 319 & 4.13755 & 4.13412 \\
36 & 3 & 7.15 & 307 & 322 & 5.09411 & 5.07093 \\
46 & 3 & 7.15 & 307 & 322 & 5.09411 & 5.09902 \\
57 & 3 & 7.27 & 307 & 322 & 5.17844 & 5.18094 \\
67 & 3 & 7.27 & 307 & 322 & 5.17844 & 5.18094 \\
76 & 3 & 7.27 & 307 & 322 & 5.17844 & 5.12989 \\
83 & 3 & 7.27 & 307 & 322 & 5.17844 & 5.23148 \\
100 & 3 & 7.27 & 307 & 322 & 5.17844 & 5.18094
\end{tabular}


A

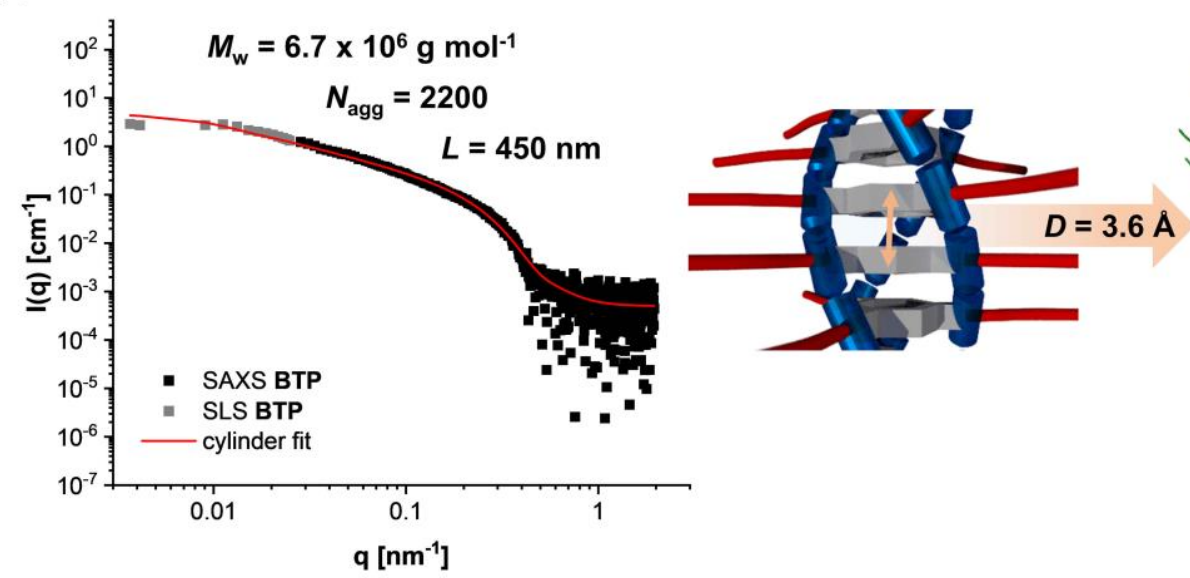

B

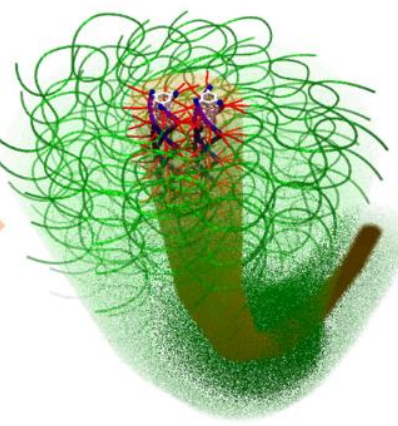

Figure S30: (A) Scattering profile of B TP directly dissolved in water, obtained by SLS (gray squares) and SAXS (black squares) and a cylindrical fit (red line) of the combined data $\left(c=1.5 \mathrm{mg} \mathrm{mL}^{-1}\right)$. Fitting parameter: $R_{\mathrm{cyl}}=6.3 \pm 1.97 \mathrm{~nm}, L_{\mathrm{cyl}}=451.97 \mathrm{~nm}$, $N=1.01557 \times 10^{-3}, S L D=1.0118 \times 10^{-3}$. (B) Proposed arrangement of BTP molecules in the cross section assuming a stacking distance of $3.6 \AA$ between the BTP cores. For further experimental details of these measurements and calculation, the reader is

\section{References}

263 1. Gruschwitz, F. V.; Fu, M.-C.; Klein, T.; Takahashi, R.; Higashihara, T.; 264 Hoeppener, S.; Nischang, I.; Sakurai, K.; Brendel, J. C., Macromolecules 2020, 53 265 (17), 7552-7560.

266 2. Klein, T.; Ulrich, H. F.; Gruschwitz, F. V.; Kuchenbrod, M. T.; Takahashi, R.; 267 Fujii, S.; Hoeppener, S.; Nischang, I.; Sakurai, K.; Brendel, J. C., Polym. Chem. $2682020,11(42), 6763-6771$. 